\title{
1 Genetic risk factors have a substantial impact on healthy life years
}

2 Sakari Jukarainen ${ }^{1 *}$, Tuomo Kiiskinen ${ }^{1,2,3}$, Aki S. Havulinna ${ }^{1,2}$, Juha Karjalainen ${ }^{1,3,4}$,

3 Mattia Cordioli ${ }^{1}$, Joel T. Rämö ${ }^{1}$, Nina Mars ${ }^{1}$, FinnGen, Kaitlin E. Samocha ${ }^{3,5}$, Hanna M.

4 Ollila ${ }^{1,3,5,6}$, Matti Pirinen ${ }^{1,8,9}$, Andrea Ganna ${ }^{1,3,4 *}$

$6{ }^{1}$ Institute for Molecular Medicine Finland (FIMM), University of Helsinki, Helsinki, 7 Finland.

$8 \quad{ }^{2}$ Finnish Institute for Health and Welfare, Helsinki, Finland.

$9{ }^{3}$ Program in Medical and Population Genetics, Broad Institute of MIT and Harvard, 10 Cambridge, MA, USA.

$11{ }^{4}$ Analytic and Translational Genetics Unit, Massachusetts General Hospital, Boston, 12 MA, USA.

$13{ }^{5}$ Center for Genomic Medicine, Massachusetts General Hospital, Boston, MA, USA.

$14{ }^{6}$ Anesthesia, Critical Care, and Pain Medicine, Massachusetts General Hospital and 15 Harvard Medical School, Boston, MA, USA.

$16{ }^{8}$ Department of Public Health, University of Helsinki, Helsinki, Finland.

$17{ }^{9}$ Helsinki Institute for Information Technology HIIT and Department of Mathematics and

18 Statistics, University of Helsinki, Helsinki, Finland.

$19{ }^{*}$ Corresponding authors:

20 Dr. Andrea Ganna and Dr. Sakari Jukarainen

21 Institute for Molecular Medicine Finland (FIMM), University of Helsinki, Helsinki, Finland

22 andrea.ganna@helsinki.fi

23 sakari.jukarainen@helsinki.fi 


\section{Abstract}

25 The impact of genetic variation on overall disease burden has not been

26 comprehensively evaluated. Here we introduce an approach to estimate the effect of

27 different types of genetic risk factors on disease burden quantified through disability-

28 adjusted life years (DALYs, "lost healthy life years"). We use genetic information from

29735,748 individuals with registry-based follow-up of up to 48 years. At the individual

30 level, rare variants had higher effects on DALYs than common variants, while common

31 variants were more relevant for population-level disease burden. Among common

32 variants, rs3798220 (LPA) had the strongest effect, with 1.18 DALYs attributable to

33 carrying 1 vs 0 copies of the minor allele. Belonging to top $10 \%$ vs bottom $90 \%$ of a

34 polygenic score for multisite chronic pain had an effect of 3.63 DALYs. Carrying a

35 deleterious rare variant in $L D L R, M Y B P C 3$, or $B R C A 1 / 2$ had an effect of around 4.1-

36 13.1 DALYs. The population-level disease burden attributable to some common variants

37 is comparable to the burden from modifiable risk factors such as high sodium intake and

38 low physical activity. Genetic risk factors can explain a sizeable number of healthy life

39 years lost both at the individual and population level, highlighting the importance of

40 incorporating genetic information into public health efforts.

43 Results of the study can be explored at: https://dsge-lab.shinyapps.io/daly genetics/ 


\section{Introduction}

45 Genome-wide association studies (GWAS) have identified thousands of variants

46 associated with biological traits and diseases ${ }^{1}$. Overall, these results demonstrate

47 widespread pleiotropy (i.e., genetic variants associated with more than one trait) ${ }^{2}$.

48 Studies commonly quantify the impact of genetic variation on a single disease at a

49 time $^{3-5}$, or when considering multiple diseases ${ }^{6-8}$ do not use a single metric that can

50 capture overall disease burden. It is therefore challenging to assess the impact of

51 genetic variation on overall health and to compare the total impact of different variants.

52 Past efforts in comparative risk assessment involve quantifying the effects of modifiable

53 adverse exposures (e.g., sodium intake) on health outcomes to inform public health

54 measures $^{9}$. For genetic risk factors this type of assessment has not been systematically

55 performed, perhaps owing to lack of interventions. However, advances in human

56 genetics (e.g., polygenic scores, PGS) have generated increasing interest in using an

57 individual's genetic risk in prioritization of screening (e.g., for cancers) and primary

58 prevention (e.g., for coronary artery disease) ${ }^{7,10-12}$, making information on genetic risk

59 clinically actionable. Also, genetic risk factors are becoming modifiable as PGSes have

60 been controversially evaluated for their potential in embryo selection ${ }^{13-15}$ and in vivo

61 gene editing is progressing towards clinical application ${ }^{16-18}$. Thus, there is a need for a

62 comparative risk assessment framework of genetic risk factors.

63 One prominent metric for disease burden is the disability-adjusted life year (DALY).

64 DALYs represent the loss of healthy life years through worsened quality of life and 65 premature death attributable to a disease ${ }^{19}$. Combining both quality of life and mortality

66 into a single metric, DALYs are used to monitor disease burden across hundreds of 3 
67 countries in the Global Burden of Disease study (GBD) ${ }^{9,19}$. The GBD estimates the 68 yearly amount of DALYs in each country attributable to a list of collectively exhaustive

69 and mutually exclusive diseases and injuries ${ }^{19}$. DALYs are the sum of years lived with

70 disability (YLDs, "lowered quality of life") and years of life lost (YLLs, "premature death")

71 (Extended Data Fig. 1). Simplifying, the yearly YLDs are estimated by multiplying the

72 prevalence of a disease by its disability weight, which represents the magnitude of

73 health loss due to living with the disease scaled between 0 (perfect health) and 1

74 (death). Yearly YLLs are estimated by multiplying the number of deaths attributable to a

75 disease by the standard life expectancy at age of death ${ }^{19}$.

76 We propose a new approach for combining genetic association results for 80 diseases

77 from two biobank studies with DALY estimates from the 2019 GBD study ${ }^{19}$ to provide an

78 overview of the impact of genetic variation on lost healthy life years both at individual

79 and population level. We rank different genetic risk factors in terms of their total health

80 impact and compare genetic risk factors with traditional modifiable risk factors,

81 presenting a template for comparative risk assessment of genetic risk factors. 


\section{Results}

\section{Estimating attributable DALYs}

84 Our method is similar to the GBD approach which estimates the disease burden

85 attributable to modifiable risk factors/exposures ${ }^{9}$ except here we consider different 86 classes of genetic risk factors: common variants, rare deleterious variants, HLA alleles,

87 APOE haplotypes, and PGSes (Fig. 1), referred to as genetic exposures. An advantage 88 over the GBD risk factors approach ${ }^{9}$ is that our genetic association results are 89 estimated using individual-level data from two large population-based biobank studies:

90 FinnGen $(n=309,136)$ and UK Biobank (UKB, $n=426,612)$ with registry-based follow-up

91 of 48.7 and 22.4 years respectively. Also, with some important caveats, the estimates

92 we present allow for a causal interpretation by virtue of genetic exposures having fewer

93 possible confounders than modifiable risk factors. In total we considered 80

94 noncommunicable diseases that account for $83.1 \%$ of the total DALYs out of all 95 noncommunicable diseases in Finland $2019^{19}$ see Supplementary Tables 2 and 3 96 (ST2-ST3).

97 For each genetic exposure-disease pair, we estimated the hazard ratio (HR) using a

98 Cox proportional hazards model. Because a single genetic variant is expected to be

99 associated with only a minority of the considered 80 diseases, we used a shrinkage

100 approach with a spike-and-slab type prior distribution for the log-HRs of each genetic 101 exposure for the 80 diseases (Methods). We discarded any genetic exposure-disease 102 associations where the posterior probability of the null model was above $10 \%$. 
medRxiv preprint doi: https://doi.org/10.1101/2022.01.25.22269831; this version posted January 28, 2022. The copyright holder for this preprint (which was not certified by peer review) is the author/funder, who has granted medRxiv a license to display the preprint in perpetuity.

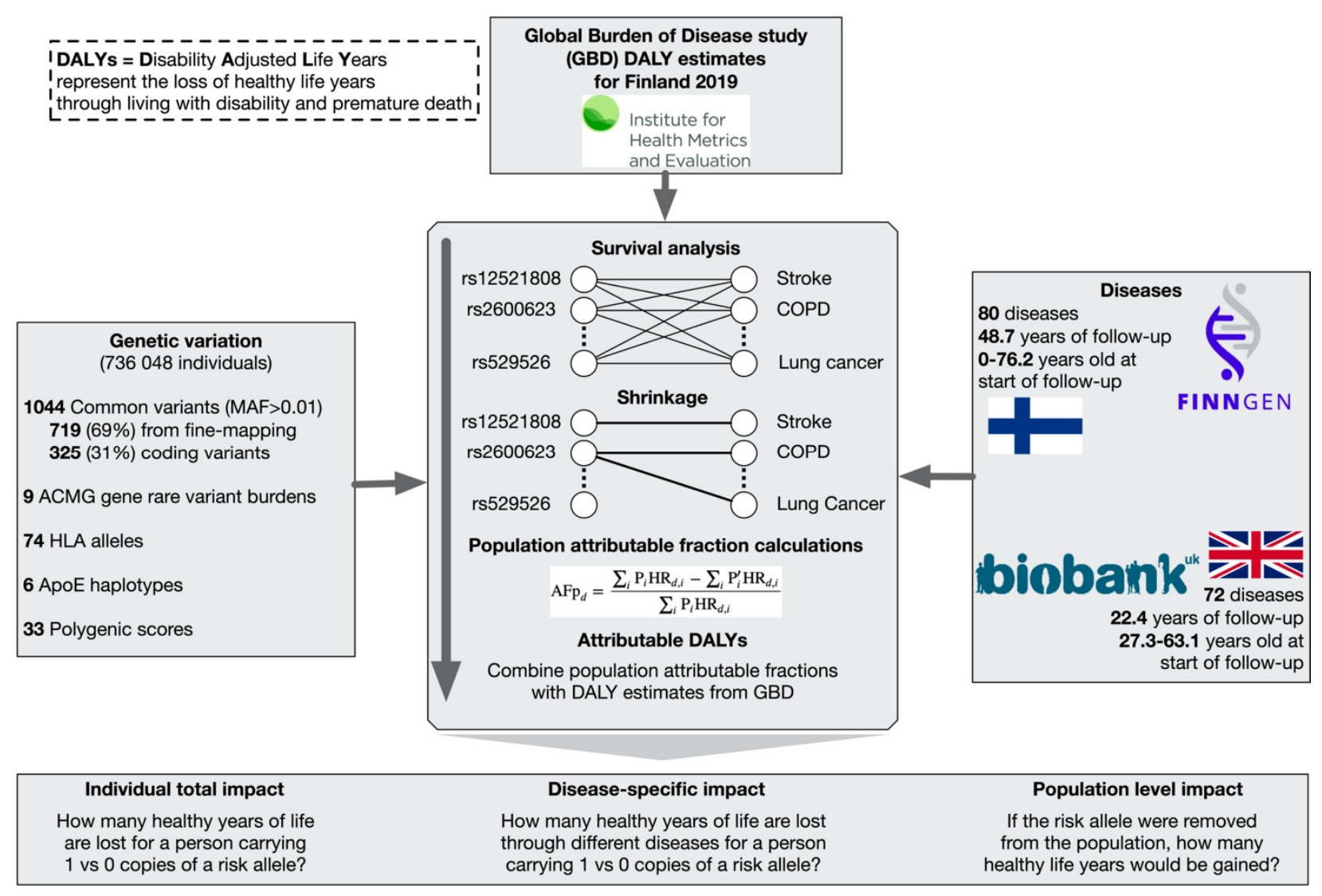

104 Fig. 1: Study overview. GBD, Global Burden of Disease study; MAF, minor allele frequency; HLA, 105 human leukocyte antigen system; ACMG, American College of Medical Genetics; AFp, 106 population attributable fraction; HR, hazard ratio.

107 Overall, we estimated the HRs through 92,800 survival analyses (associations of 1044 108 common variants, 9 rare variant burdens, 74 HLA alleles, and 30 PGSes with 80 109 diseases) and, after shrinkage, retained 3,123 HRs for genetic exposure-disease pairs, 110 most of which (67.1\%) were genome-wide significant $\left(P<5 \times 10^{-8}\right)$ and $99 \%$ had an 111 association with $P<7.3 \times 10^{-4}$ (Extended Data Fig. 2). Using the HR estimates and 112 frequencies of the genetic exposures, we estimated the population attributable fraction 113 of disease cases for each genetic exposure (the proportion of disease cases that would 114 be prevented if the exposure was removed). We combined these fractions with disease- 
medRxiv preprint doi: https://doi.org/10.1101/2022.01.25.22269831; this version posted January 28, 2022. The copyright holder for this preprint (which was not certified by peer review) is the author/funder, who has granted medRxiv a license to display the preprint in perpetuity.

It is made available under a CC-BY 4.0 International license .

115 specific population DALYs for Finland 2019 from the GBD $^{19}$ (ST3) to obtain attributable

116 DALY estimates (Methods). Finally, we summed attributable DALYs across the 80

117 diseases to estimate the total impact of genetic exposures. The total individual

118 attributable DALYs, our main measure of interest, can be interpreted as the expected

119 loss of healthy life years for an individual attributable to having a certain genetic

120 exposure at birth. Because we consider different types of genetic exposures the exact

121 definition of attributable DALYs varies by exposure type. Because the disease

122 definitions in GBD are not overlapping and the DALYs are comorbidity-corrected ${ }^{19}$, the

123 final estimates take double-counting into account.

124 Attributable DALYs for common variants

125 For all variants, we defined the minor allele as the less common allele in FinnGen. We 126 considered 1,044 independent common variants (minor allele frequency, MAF $>1 \%$ ).

127 We selected 564 of these based on having at least one $P<5 \times 10^{-8}$ association with any

128 of the 80 diseases and having the highest probability of being causal within a SuSiE

129 fine-mapped ${ }^{20} 95 \%$ credible set in FinnGen. Additionally, we selected 155 common

130 variants with at least one $P<5 \times 10^{-12}$ association with 6 traditional risk factor traits (BMI,

131 HbA1c, HDL cholesterol, LDL cholesterol, systolic blood pressure, cigarettes per day)

132 that had the highest probability of being causal within a within a SuSiE fine-mapped

$13395 \%$ credible set in $\mathrm{UKB}^{21}$. Last, we included 325 coding variants having a $P<5 \times 10^{-8}$

134 association with one of the diseases in FinnGen. Among the 1044 variants, $34.6 \%$ were

135 annotated as missense $(n=335)$ or putative loss-of-function ( $n=26$, pLOF). The HRs for

136 common variants were comparable between FinnGen and UKB (Extended Data Fig. 3)

137 and we consequently meta-analyzed the results. All estimates are for the comparison of 
medRxiv preprint doi: https://doi.org/10.1101/2022.01.25.22269831; this version posted January 28, 2022. The copyright holder for this preprint (which was not certified by peer review) is the author/funder, who has granted medRxiv a license to display the preprint in perpetuity.

It is made available under a CC-BY 4.0 International license .

1381 vs 0 copies of the minor allele, so the individual attributable DALYs thus correspond to

139 the expected loss of healthy life years if an individual with 0 copies of the minor allele

140 had instead 1 copy at birth.

141 Overall, carrying 1 vs 0 copies of the common variants we studied resulted in relatively

142 small effects on lost healthy life years in terms of DALYs, with only 56 out of 1,044

$143(5.4 \%)$ variants having over 0.25 attributable DALYs (ST11-ST12). Many of the top hits

144 for attributable DALYs were in chromosome 6, both inside and outside of the HLA

145 region (Fig. 2a). We imputed the 7 classical HLA genes at the two-field resolution level

146 (i.e., unique protein sequence level) using a Finnish-specific reference panel ${ }^{22}$ and

147 provide attributable DALYs for HLA alleles in (Extended Data Fig. 4, ST9). However,

148 we caution about the interpretation of attributable DALYs in this context because, for

149 multiallelic loci, the estimates are not straightforward to interpret.

150 The variant with the highest number of attributable DALYs was rs3798220, a missense

151 variant in the $L P A$ gene, with 1.18 (95\% confidence interval $(\mathrm{Cl}) 1.03-1.32, P=1.3 \times 10^{-58}$ )

152 attributable DALYs from carrying 1 vs 0 copies of the $C$ allele (Fig. 2B). The effect was

153 almost exclusively through ischemic heart disease (1.11 DALYs) and to a lesser extent

154 through non-rheumatic valvular heart disease (0.046 DALYs) and lower extremity

155 peripheral artery disease (0.016 DALYs) despite similar risk increases. This is because

156 of the larger number of population DALYs attributed to ischemic heart disease by the

157 GBD (e.g., 60-fold difference to lower extremity peripheral artery disease, ST3),

158 illustrating the importance of using measures other than just relative risk. 
medRxiv preprint doi: https://doi.org/10.1101/2022.01.25.22269831; this version posted January 28, 2022. The copyright holder for this preprint (which was not certified by peer review) is the author/funder, who has granted medRxiv a license to display the preprint in perpetuity.

It is made available under a CC-BY 4.0 International license.

a

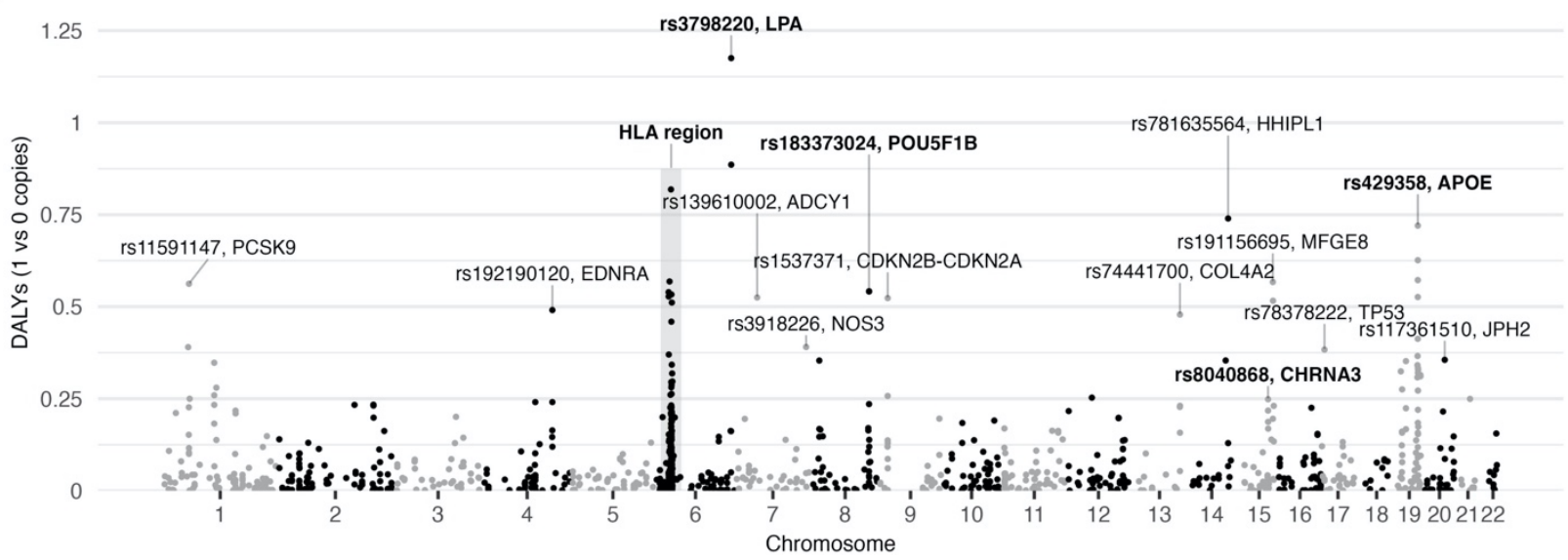

b

rs3798220, LPA

c
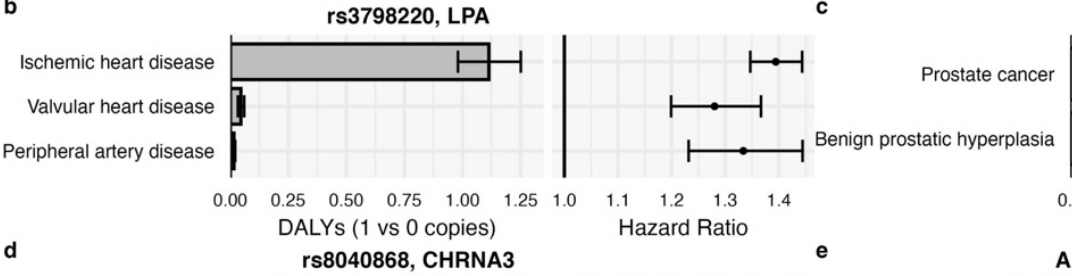

rs183373024, POU5F1B
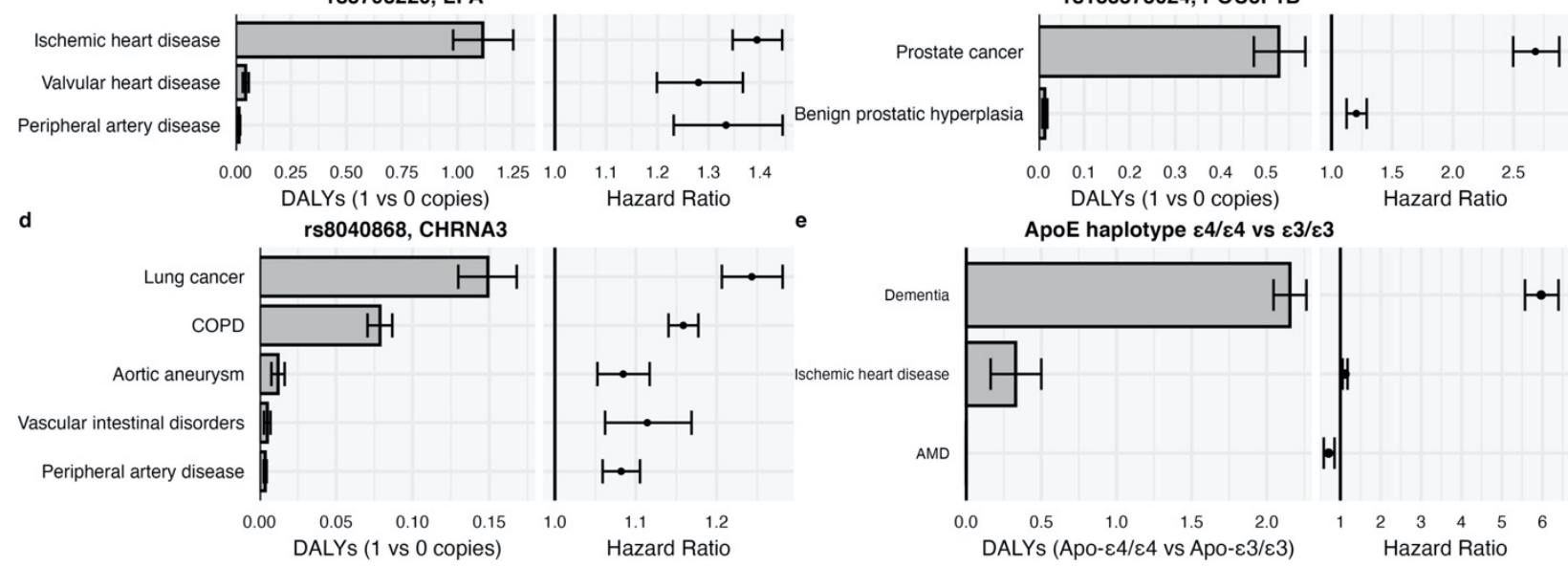

$\begin{array}{llllll}0.0 & 0.1 & 0.2 & 0.3 & 0.4 & 0.5\end{array}$ DALYs ( 1 vs 0 copies)

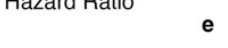

ApoE haplotype $\varepsilon 4 / \varepsilon 4$ vs $\varepsilon 3 / \varepsilon 3$
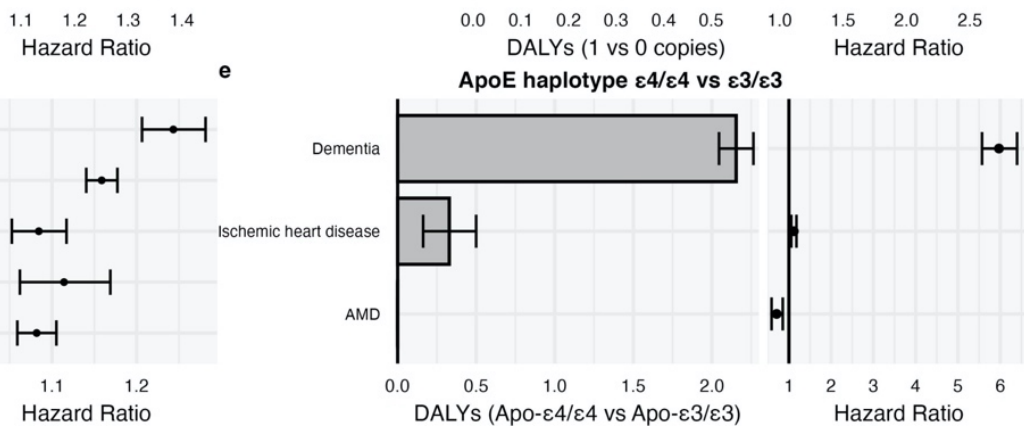

Fig. 2: Effect of common variants on DALYs. a, Absolute effect on DALYs from carrying 1 vs 0

copies of the minor allele for each common variant. We analyzed separately imputed alleles in the HLA region. Results for this region are provided in (Extended Data Fig. 4). b-e, For 3 common variants and the APOE haplotypes (in bold in panel a) we reported attributable DALYS and HRs separately for each disease. Error bars denote $95 \%$ confidence intervals. COPD, chronic obstructive pulmonary disease; AMD, age-related macular degeneration.

166 One interesting example is rs183373024, a non-coding variant near the POU5F1B 167 gene ${ }^{23}$, with $0.54\left(95 \% \mathrm{Cl} 0.48-0.60, P=1.1 \times 10^{-74}\right)$ attributable DALYs mainly through 168 prostate cancer (Fig. 2c). Another example is rs8040868, a synonymous variant in the well-known CHRNA5/A3/B4 gene cluster associated with nicotine dependence ${ }^{24}$, with

$170 \quad 0.25\left(95 \% \mathrm{Cl} 0.23-0.27, P=1.6 \times 10^{-95}\right)$ attributable DALYs (Fig. 2d), with effects through 
171 lung cancer, COPD, aortic aneurysm, vascular intestinal disorders, and lower extremity

172 peripheral artery disease (all consequences of smoking).

173 Given the strong associations between APOE alleles and longevity ${ }^{25}$, we defined the

174 three main APOE alleles determined by two SNPs rs429358 and rs7412. Carrying the

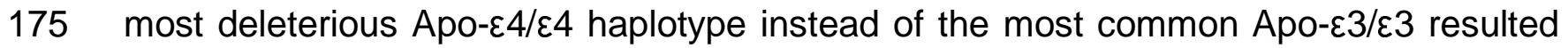

176 in $2.48\left(95 \% \mathrm{Cl} 2.28-2.68, P=1.0 \times 10^{-128}\right)$ attributable DALYs, mainly through increase in

177 risk of Alzheimer's disease and other dementias (HR=5.97, 95\% Cl 5.57-6.40, Figure

178 2e, ST8). Overall, out of the top $10 \%$ common variants with the highest number of

179 attributable DALYs, $49.4 \%$ were significantly associated (nominal $P<0.05$ ) with longevity

180 in the largest GWAS on lifespan ${ }^{5}$ versus $18 \%$ in the bottom $10 \%$.

181 The full results for the common variant analysis can be explored at: https://dsge-

182 lab.shinyapps.io/daly genetics/

\section{Attributable DALYs for rare deleterious variants}

184 Rare deleterious coding variants $(\mathrm{MAF}<0.001)$ are often clinically relevant because of

185 their large individual (not population) level effects. However, past studies quantifying the

186 effect of rare variants have relied on highly selected clinical populations due to lack of

187 population-based whole-exome/genome sequencing data. Recent whole-exome

188 sequencing data from UKB $(n=174,379)$ provides a unique opportunity to address this

189 issue.

190 The American College of Medical Genetics and Genomics (ACMG) recommend

191 reporting incidental findings in clinical exome and genome sequencing for 73 genes $^{26,27}$.

192 We estimated the attributable DALYs for two types of burdens for these ACMG genes: 
193 1) putative loss-of-function (pLOF) variant burden and 2) "pathogenic" or "likely

194 pathogenic" Clinvar ${ }^{28}$ variant burden (for BRCA1/2 we used "pathogenic" ENIGMA ${ }^{29}$

195 variants instead). We report results for genes with at least 35 individuals with a positive

196 burden and at least one retained burden-disease association. Taking the pLOF burdens

197 as an example, the estimated individual attributable DALYs correspond to the expected

198 loss of healthy life years if an individual carrying no loss-of-function variants in the gene

199 would be instead carrying at least one.

200 The 5 genes most impactful in terms of DALYs (Fig. 3, ST15-16) were LDLR (ischemic 201 heart disease), BRCA2 (breast, ovarian, liver, and prostate cancer, COPD), MYBPC3 202 (cardiomyopathy and myocarditis), BRCA1 (breast and ovarian cancer), and MLH1 203 (colon and rectum cancer). As an example, individuals carrying one pLOF in BRCA1 204 lose on average $4.08\left(95 \% \mathrm{Cl} 2.74-6.32, P=1.4 \times 10^{-5}\right)$ healthy life years through breast 205 cancer $(\mathrm{HR}=7.01,95 \% \mathrm{Cl}$ 4.94-9.94, DALYs=2.11, 95\% Cl 1.39-3.14) and ovarian 206 cancer $(\mathrm{HR}=16.2,95 \% \mathrm{Cl}$ 8.22-31.8, DALYs=1.97, 95\% Cl 0.95-3.93). 


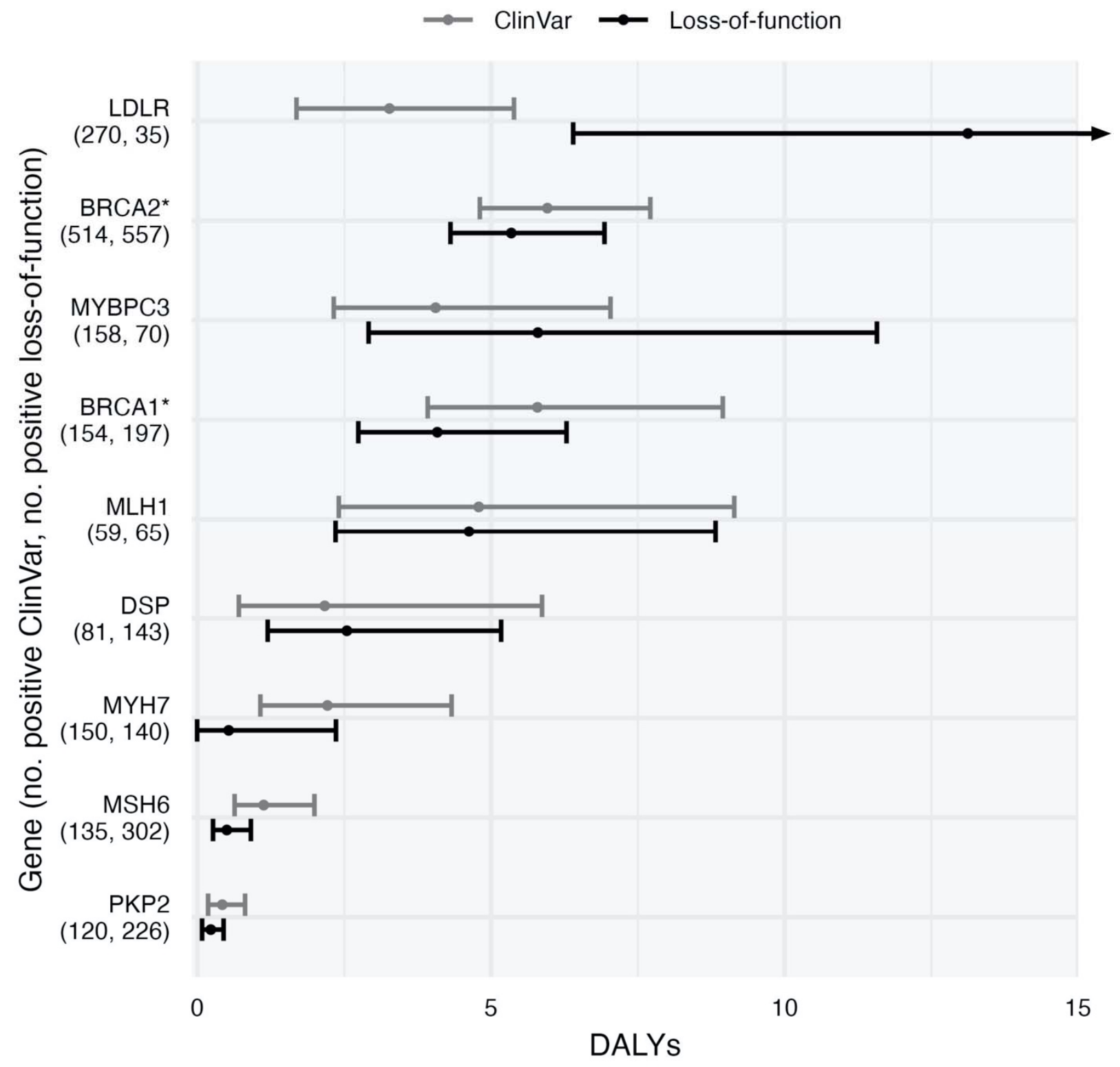

208 Fig. 3: DALYs attributable to carrying a deleterious rare variant in ACMG genes. The ClinVar ${ }^{28}$

209 burden contains all variants annotated as "pathogenic" or "likely pathogenic". *For BRCA1 and 210 BRCA2 we only considered variants from ENIGMA ${ }^{29}$ annotated as "pathogenic". The loss-of-

211 function burden contains all variants annotated as putative loss-of-function with high 212 confidence in gnomAD ${ }^{30}$. Error bars denote $95 \%$ confidence intervals. 
medRxiv preprint doi: https://doi.org/10.1101/2022.01.25.22269831; this version posted January 28, 2022. The copyright holder for this preprint (which was not certified by peer review) is the author/funder, who has granted medRxiv a license to display the preprint in perpetuity.

It is made available under a CC-BY 4.0 International license .

\section{Attributable DALYs for polygenic scores}

215 We considered 30 PGSes covering major diseases, clinical risk factors, and

216 psychobehavioral traits. We estimated the effect on DALYs attributable to belonging to

217 the top $10 \%$ of a PGS versus the bottom $90 \%$, which depict the expected loss of healthy

218 life years if an individual in the bottom $90 \%$ of a PS was instead in the top $10 \%$ at birth.

219 Overall, the attributable DALYs varied from 0.07 (inflammatory bowel disease ${ }^{31}$ ) to 3.81

220 (shorter lifespan ${ }^{32}$ ) (Fig. 4a, ST13-ST14). Many of the PGSs exhibited significant

221 pleiotropy, with a median of 16 (IQR 9-28) PGS-disease associations remaining after

222 shrinkage. PGSes with the highest number of retained disease associations were

223 multisite chronic pain ${ }^{33}(n=44)$, lower educational attainment ${ }^{34}(n=40)$, and shorter

$224 \operatorname{lifespan}^{32}(\mathrm{n}=40)$.

225 A PGS predicting shorter lifespan ${ }^{8}$ had the highest impact. Individuals in the top $10 \%$ of 226 the PGS are expected to lose $3.81\left(95 \% \mathrm{Cl} 3.52-4.13, P=4.6 \times 10^{-131}\right)$ healthy life years 227 compared to an individual in the bottom 90\%. This PGS acts mainly through ischemic 228 heart disease (1.2 DALYs) and to lesser extent through lung cancer, ischemic stroke, 229 COPD, type 2 diabetes, substance use disorders and low back pain, each accounting 230 between 0.21-0.26 DALYs (Fig. 4b). Interestingly, a PGS for multisite chronic pain ${ }^{33}$ 231 had the second highest impact at $3.63\left(95 \% \mathrm{Cl} 3.33-3.93, P=5.0 \times 10^{-124}\right)$ DALYs, mainly 232 through low back pain (0.48 DALYs), ischemic heart disease (0.47), substance use 233 disorder (0.37), COPD (0.23), depression (0.22), and neck pain (0.21) (Fig. 4c). Results 234 for the polygenic score analysis can be explored at: https://dsge235 lab.shinyapps.io/daly genetics/ 
a

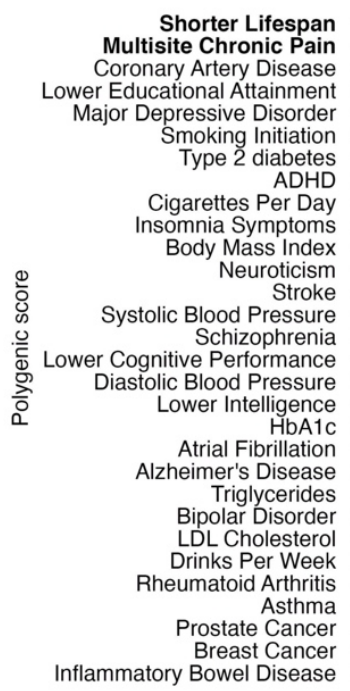

b

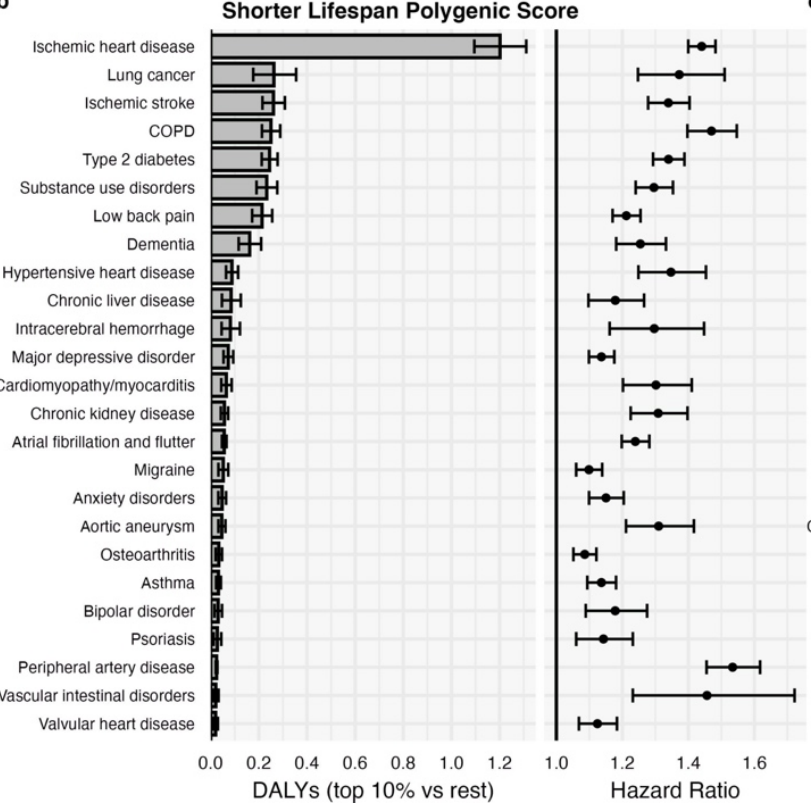
Attributable DALYs for Polygenic Scores

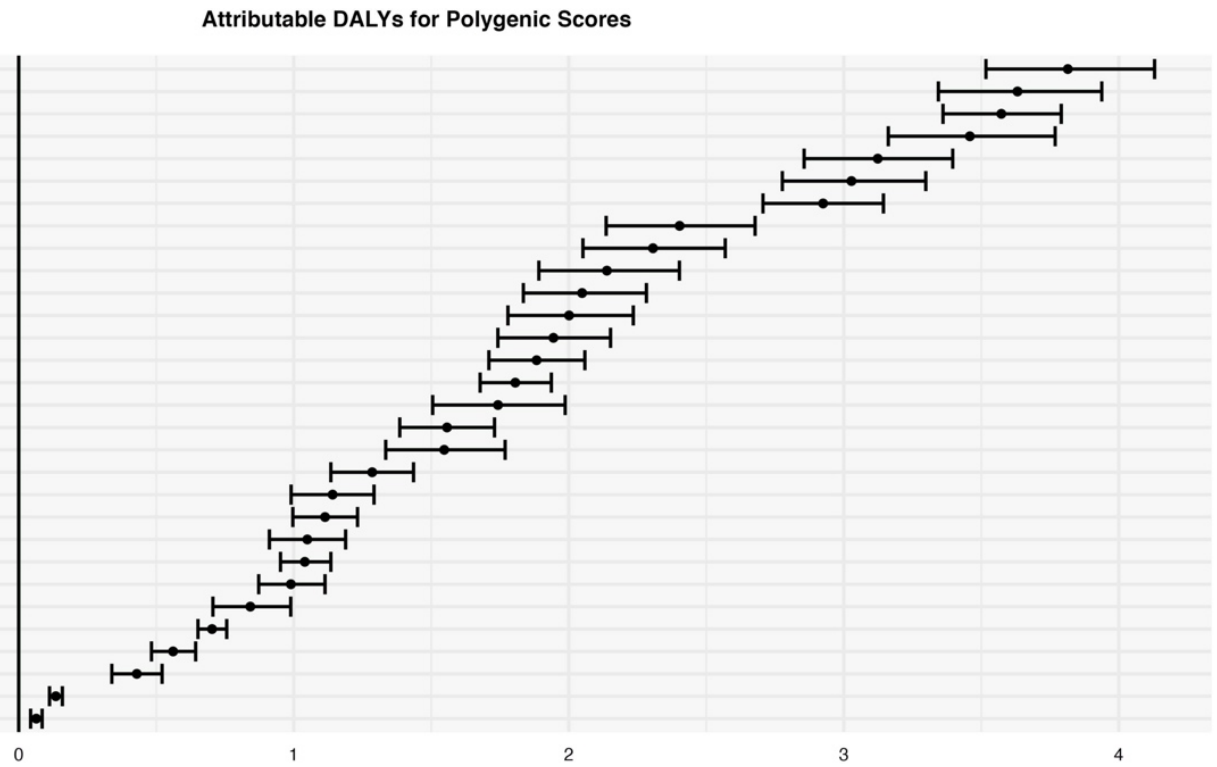

DALYs (top $10 \%$ vs rest)

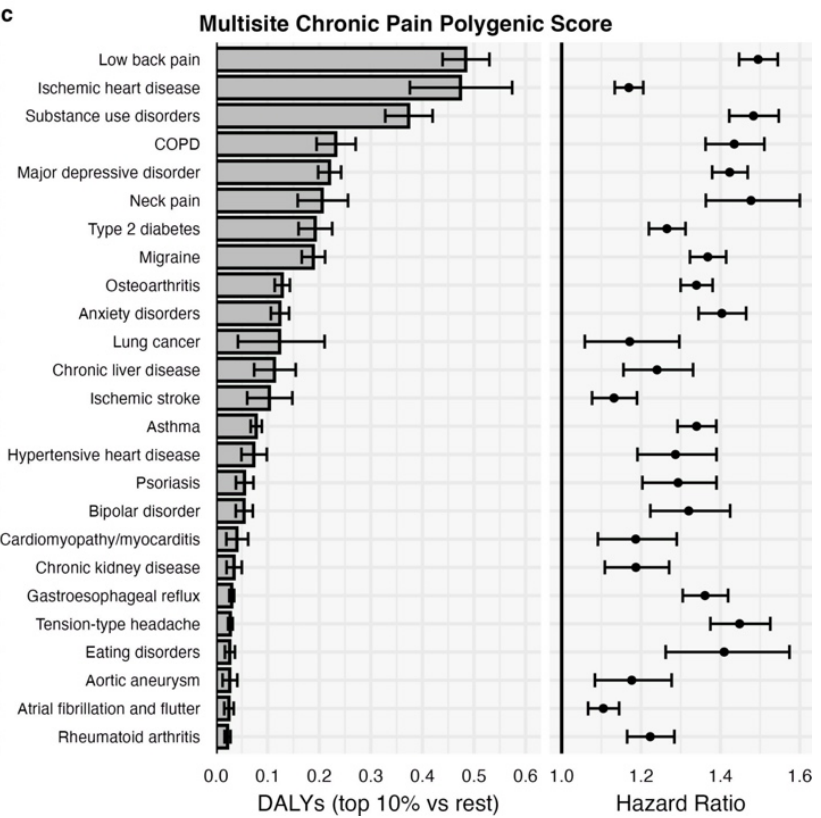

237 Fig. 4: Polygenic score impact on DALYs. a, DALYs attributable to belonging in the top $10 \%$ vs

238 bottom $90 \%$ of each PGS. b, c, Top 25 diseases in terms of attributable DALYs and HRs for two 239 PGSes (bold in panel a). Error bars denote 95\% confidence intervals. ADHD, attention deficit 240 hyperactivity disorder; HbA1c, glycated hemoglobin. 
medRxiv preprint doi: https://doi.org/10.1101/2022.01.25.22269831; this version posted January 28, 2022. The copyright holder for this preprint (which was not certified by peer review) is the author/funder, who has granted medRxiv a license to display the preprint in perpetuity.

\section{Sex-specific effects}

243 We repeated all analyses stratified by sex and present estimates for the main analyses

244 (apart from rare variants) by sex in supplements (ST8-ST9, ST11-ST14). Significant sex

245 differences in total DALYs at $P<0.05$ were observed for $474(45 \%)$ of the common

246 variants (Fig. 5a). Sex differences in attributable DALYs can result from differences in

247 the effect of the genetic exposure on the disease (i.e., differences in HRs) or differences

248 in DALYs attributed to men and women by the GBD ${ }^{19}$. rs738409 (PNPLA3-I148M), a

249 missense variant in PNPLA3 linked to liver fat accumulation and steatohepatitis ${ }^{35}$,

250 provides a clarifying example: Carrying 1 vs 0 copies of the minor allele resulted in 0.27

$251\left(95 \% \mathrm{Cl} 0.24-0.30, P=1.6 \times 10^{-71}\right)$ attributable DALYs in males and $0.05(95 \% \mathrm{Cl} 0.03-$

$2520.07, P=2.5 \times 10^{-7}$ ) DALYs in females (sex difference $P=1.0 \times 10^{-34}$ ). The sex difference is

253 in part driven by differences in HR between men and women for chronic liver disease

254 (1.32, $95 \% \mathrm{Cl} 1.28-1.37$ in males vs $1.21,95 \% \mathrm{Cl} 1.17-1.26$ in females, $P$ for sex 255 difference $=3.6 \times 10^{-4}$ ) and, in part, because DALYs for chronic liver disease are higher 256 in men than women ${ }^{19}$ (431 vs 158 yearly DALYs per 100,000) (Fig. 5c).

2578 out of 30 PGSes had significant sex differences in attributable DALYs (Fig. 5b). Most 258 of such differences were explained by different DALYs attributed to men and women 259 rather than differences in underlying association estimates. For example, a PGS 260 predicting weekly alcohol consumption had similar HRs between sexes across all 261 diseases (Fig. 5d) but markedly different effect on DALYs for substance use disorders 262 reflecting the higher disease burden disease for substance use disorders among men 263 (1,500 yearly DALYS per 100,000) than women (497 yearly DALYs per 100,000) as 264 estimated by the $\mathrm{GBD}^{19}$. 
a

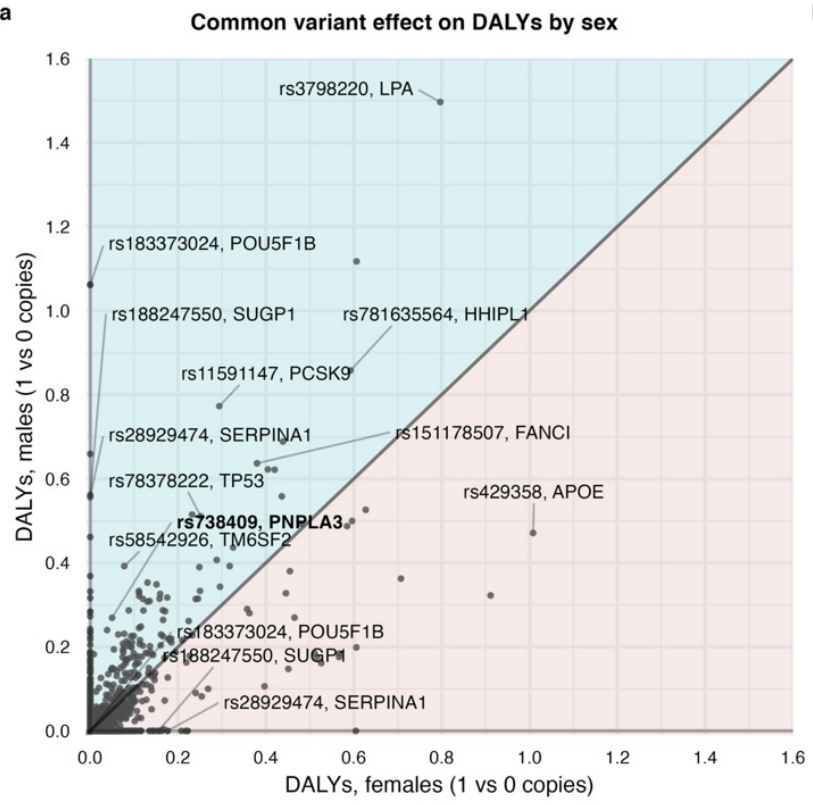

c rs738409, PNPLA3

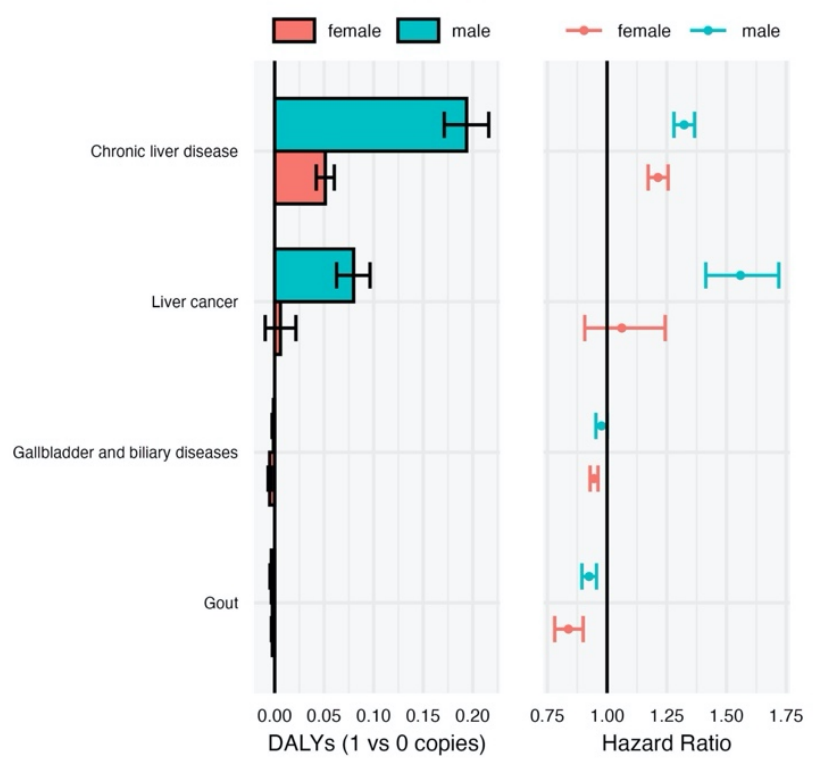

b Polygenic scores with significant sex difference

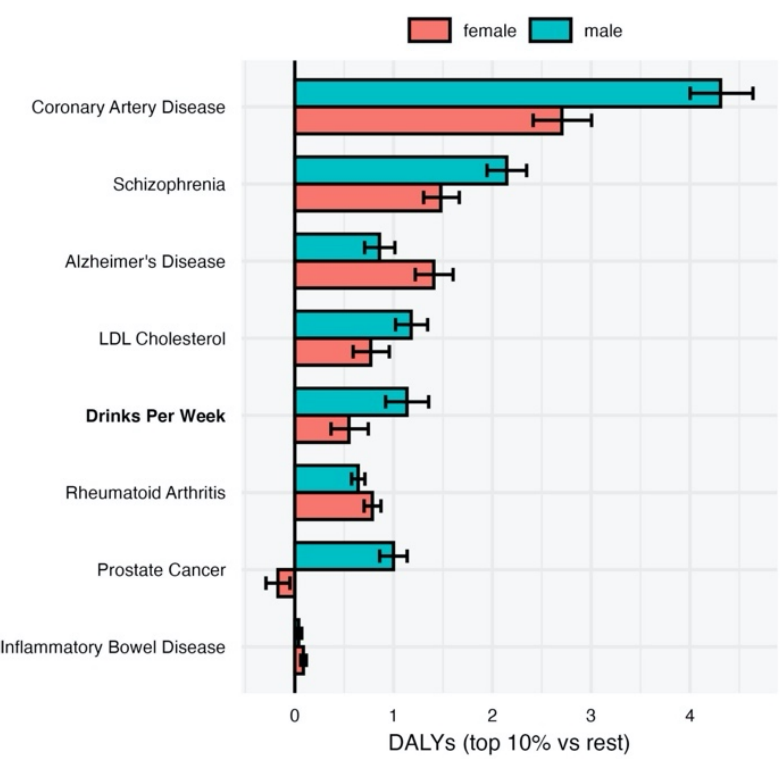

d

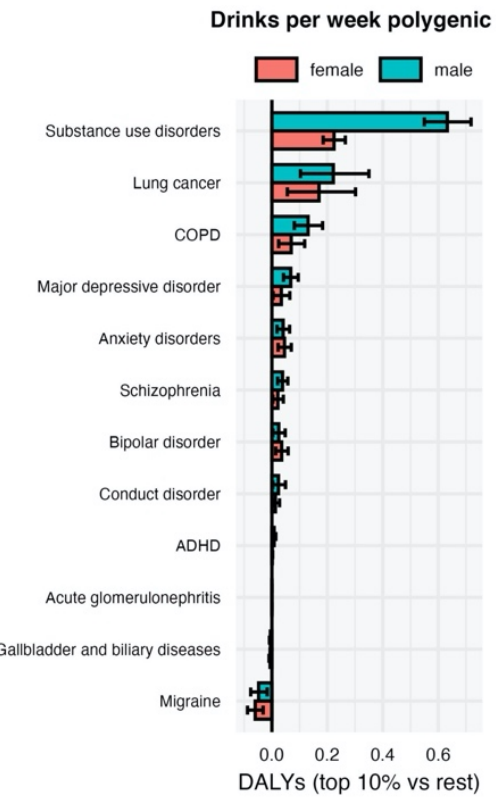

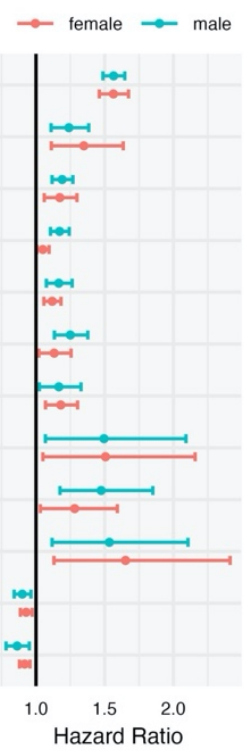

266 Fig. 5: Sex-specific impact of common variants and polygenic scores on DALYs. a, Absolute 267 attributable DALYs from carrying 1 vs 0 copies of the minor allele separately for males and 268 females. b, Polygenic scores (top 10\% vs rest) with a significant sex difference in attributable 269 DALYs. c, For rs738409 (PNPLA3) (in bold in panel a) we report the attributable DALYs and HRs 270 for each disease by sex. $d$, Attributable DALYs and HRs by disease and sex for the PS predicting 271 drinks per week (in bold in panel b). Error bars denote nominal 95\% confidence intervals. 


\section{Population attributable DALYs for common variants}

273 So far, our results have been from an individual's perspective. Next, for the Finnish

274 population, we estimated the amount of attributable population DALYs per year per

275100,000 from all (heterozygous and homozygous) carriers of the minor allele: the

276 expected amount of healthy life years per year per 100,000 individuals in the population

277 that would be gained if the minor allele were completely removed. rs7859727

278 (CDKN2B-CDKN2A) had the highest population attributable DALYs, with minor allele

279 carriers accounting for $447\left(95 \% \mathrm{Cl} 420-473, P=4.1 \times 10^{-231}\right)$ yearly population DALYs

280 per 100,000 in Finland 2019 (Fig. 6a). The large population effect of this variant is

281 explained by its effect on ischemic heart disease $(\mathrm{HR}=1.17,95 \% \mathrm{Cl} 1.16-1.18)$ and high

282 frequency in the Finnish population $(\mathrm{MAF}=41 \%)$. Comparing to population DALY

283 estimates for classic risk factors from the $\mathrm{GBD}^{9}$ (Fig. 6a), the attributable population

284 DALY estimates of several common variants are similar to the total impact of a diet high 285 in sodium (300 yearly population DALYs per 100,000), low physical activity (415), and 286 drug use (595), but substantially less impactful than the most important classic risk

287 factors such as high systolic blood pressure (3666), smoking (2992), and high BMI $288 \quad(2506)^{9}$. 
medRxiv preprint doi: https://doi.org/10.1101/2022.01.25.22269831; this version posted January 28, 2022. The copyright holder for this preprint (which was not certified by peer review) is the author/funder, who has granted medRxiv a license to display the preprint in perpetuity.

It is made available under a CC-BY 4.0 International license .

a Population attributable DALYs, common variants vs GBD risk factors

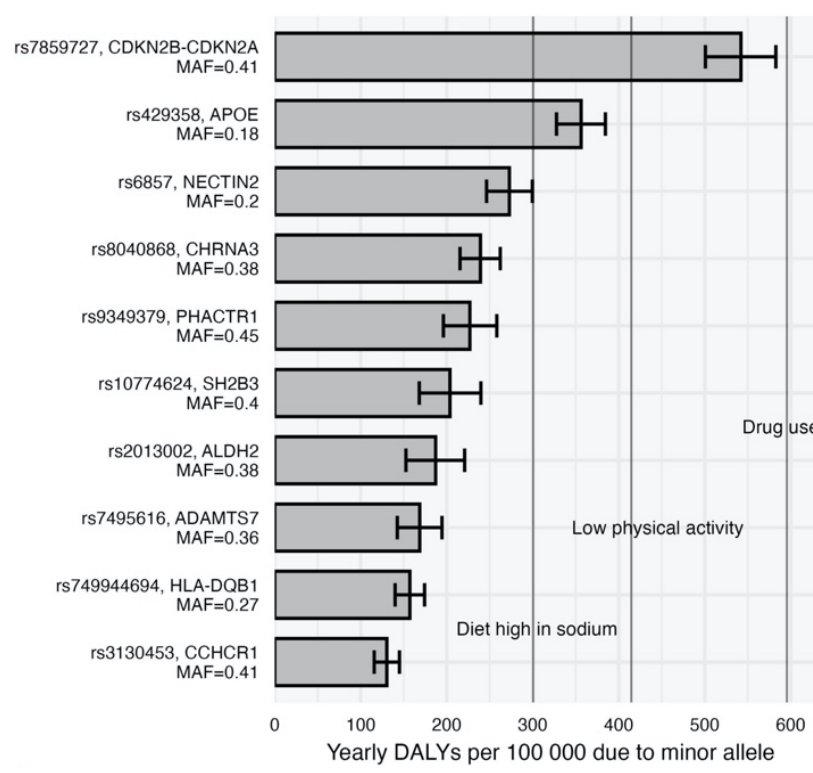

b Ischemic heart disease risk, comparison of variants and risk factors $\rightarrow$ GBD risk factor $\rightarrow$ Variant

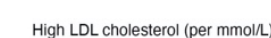

High BMI (per 5kg/m2) High systolic blood pressure (per $10 \mathrm{mmHg}$ ) rs3798220, LPA rs781635564, HHIPL1 rs191156695, MFGE8 rs11591147, PCSK9 rs139814895, FANCI rS192190120, EDNRA High fasting plasma glucose (per mmo/L) rs139610002, ADCY1 rs7859727, CDKN2B-CDKN2A IS74441700, COL4A2 rS7412, APOE

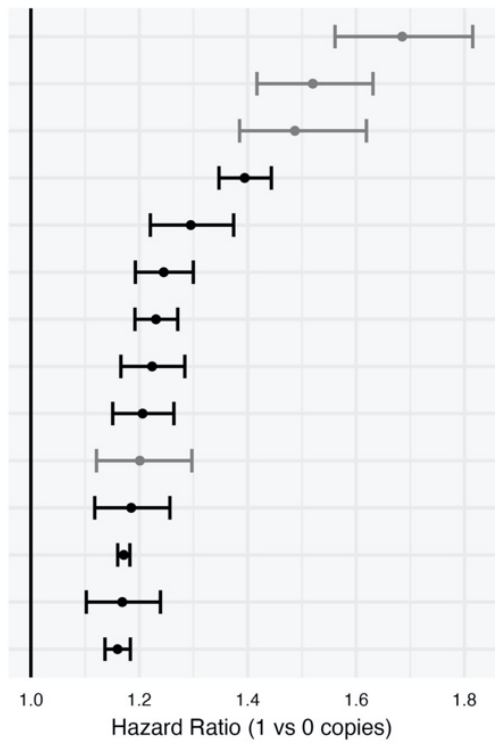

Fig. 6: Effect of common variants on population-level DALYs in the Finnish population. a, Bars represent yearly population-level DALYs attributable to top 10 ranking common variants. The vertical lines represent yearly population-level DALYs attributable to three selected classic risk factors as estimated by the $\mathrm{GBD}^{9}$ for Finland 2019. b, Top 10 HRs for ischemic heart disease of

294 common variants and 4 HRs of conventional risk factors as estimated by the GBD (50-54 year 295 age group) $)^{9}$.

296 In other words, removing the risk allele rs7859727 from the Finnish population would 297 have a comparable expected impact on increasing healthy life years as changing 298 sodium consumption or physical activity to the theoretical minimum risk level, or 299 completely removing drug use as a problem. To further expand on this concept, we 300 compared the HRs for ischemic heart disease between 8 common variants and 4 301 clinical risk factors (Fig. 6b). Clinically significant changes in traditional risk factors (e.g., $30210 \mathrm{mmHg}$ higher systolic blood pressure, $1 \mathrm{mmol} / \mathrm{L}$ higher fasting glucose) as estimated 303 by the GBD lead to comparable increases in ischemic heart disease risk (HR=1.20 to 
$3041.69)^{9}$ as having 1 vs 0 copies of the minor allele for the cardiovascular risk variants

$305(\mathrm{HR}=1.16$ to 1.39$)$.

\section{Additional population DALYs attributable to Finnish-enrichment}

307 Finland is a well-known example of an isolated population where multiple historical 308 bottlenecks ${ }^{36}$ have contributed to the enrichment of several functional genetic 309 variants $^{36,37}$ otherwise rare in non-Finnish populations. It is therefore interesting to 310 estimate the population attributable DALYs that are due to the enrichment in the Finnish

311 population compared to non-Finnish-non-Swedish-non-Estonian European (NFSEE)

312 populations. There were 56 Finnish-enriched variants that had at least 5 -fold MAF 313 enrichment in the Finnish ancestry compared to NFSEE ancestry (from gnomAd) where

314 the NFSEE MAF was below 0.01 . The largest impact on population attributable DALYs 315 (Fig. 7) was observed for rs143473297 (TOMM40) contributing to 56.1 (95\% Cl 50.1-

$31662.0, P=6.3 \times 10^{-76}$ ) yearly population DALYs per 100,000 individuals of Finnish ancestry

317 through increased risk of dementia (HR=1.95, 0.57 individual DALYs, ST12). This

318 variant had a 152-fold MAF enrichment in the Finnish population and a negligible effect

319 in NFSEE ancestry. One remarkable example of a protective Finnish-enriched variant is 320 rs191156695, an inframe insertion in MFGE $8^{38}$. The presence of the Finnish-enriched

321 allele in the population contributes to preventing $39.1\left(95 \% \mathrm{Cl} 32.0-45.8, P=4.6 \times 10^{-29}\right)$

322 yearly population DALYs per 100,000 individuals of Finnish ancestry (Fig. 7) solely

323 through decreasing ischemic heart disease risk ( $\mathrm{HR}=0.80,95 \% \mathrm{Cl} 0.77-0.84)$. 


\section{Population DALYs of Finnish enriched variants}

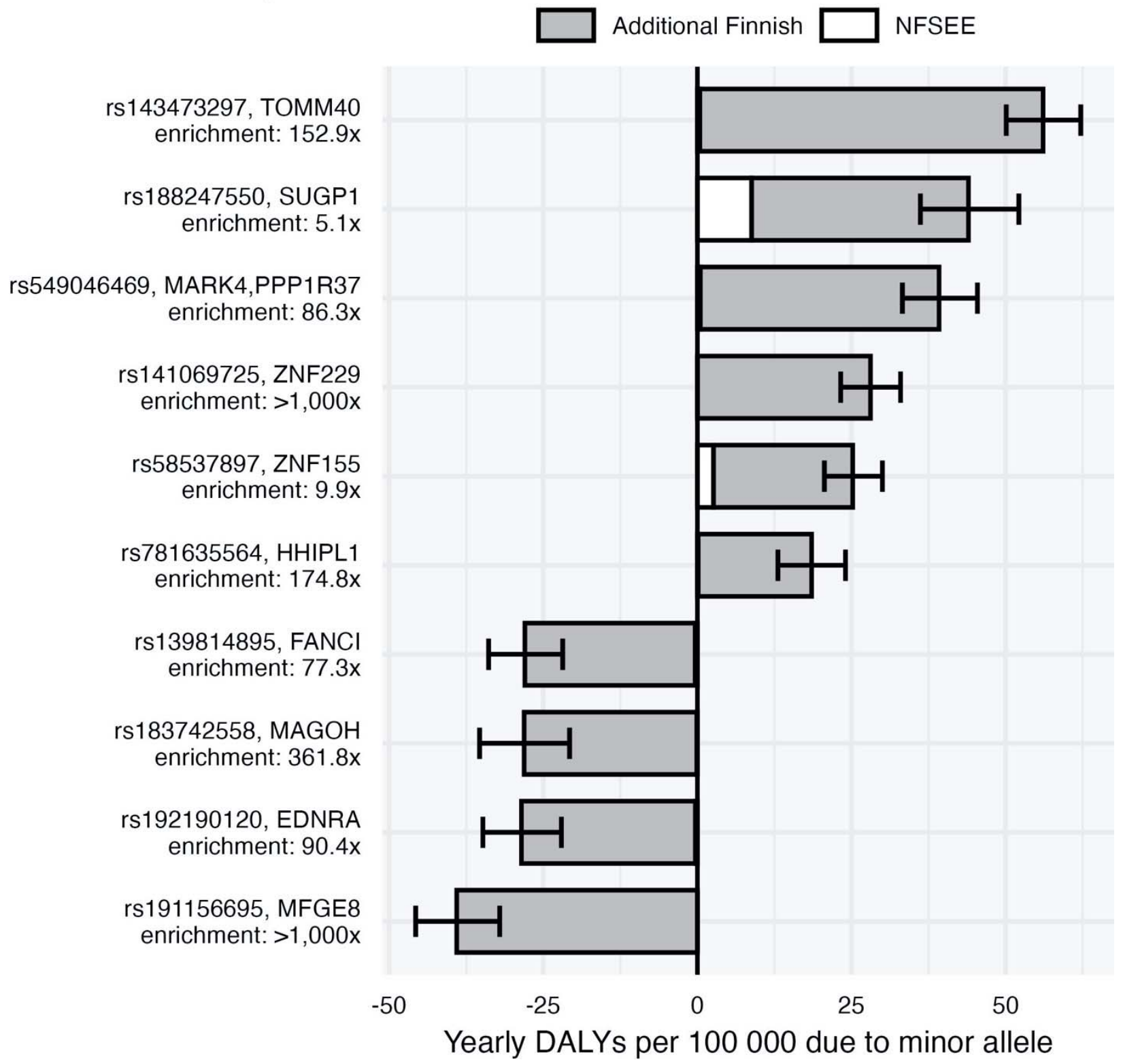

325 Fig. 7: Impact of Finnish-enriched variants on population-level DALYs. Proportion of disease-

326 specific population DALYs accounted for by the 9 Finnish-enriched variants with the largest

327 effects. The white part of the bars denotes the number of population DALYs attributable to the

328 variant if the Finnish MAF were equal to the NFSEE MAF, the grey part is the additional 329 population DALYs resulting from the enrichment in the Finnish population. Error bars denote 330 nominal 95\% confidence intervals. NFSEE, non-Finnish-non-Swedish-non-Estonian Europeans.

331 For many variants the contribution in NFSEE is neglectable and white bar not visible. 
medRxiv preprint doi: https://doi.org/10.1101/2022.01.25.22269831; this version posted January 28, 2022. The copyright holder for this preprint (which was not certified by peer review) is the author/funder, who has granted medRxiv a license to display the preprint in perpetuity.

It is made available under a CC-BY 4.0 International license.

\section{Sensitivity analyses}

333 We performed three main sensitivity analyses. First, we explored whether the effect of

334 the genetic exposures on the diseases was age dependent by performing age-stratified

335 survival analyses. Perhaps unsurprisingly ${ }^{39}$, we observed age-varying HRs for genetic

336 exposures (Extended Data Fig. 5). For example, for a coronary artery disease PGS ${ }^{40}$,

337 the HRs were $2.50(95 \% \mathrm{Cl} 2.31-2.70)$ for the $50-54$ age group and $1.75(95 \% \mathrm{Cl} 1.62-$

338 1.87) for the 70-74 age group.

339 Second, we examined if accounting for relatedness would result in different estimates

340 for a subset of the survival analyses. For 2562 common variants-disease pairs we

341 estimated the HRs using a survival model clustered by family indicator to generate

342 robust standard errors. Compared to the main analysis estimates, robust standard

343 errors were median 1.0128 (IQR: 1.0044-1.0205) times larger. Thus, accounting for

344 relatedness would not meaningfully affect the confidence intervals and $P$-values.

345 Third, we examined the performance of our shrinkage approach, showing that it is

346 robust to the choice of different prior parameters (Extended Data Fig. 6) and can

347 identify true causal variants in simulated GWAS data (Extended Data Fig. 7, ST7). We

348 further compare our shrinkage approach with a conservative colocalization-based

349 approach for 48 of the diseases (Supplementary Information). The colocalization

350 approach identified 94 common variants for which two or more diseases co-localize.

$35182.0 \%$ of these variants and the corresponding colocalizing diseases were identified by

352 our shrinkage approach. Conversely, only $22.4 \%$ of pairs of diseases reported by our

353 approach were also found in the colocalization analysis. This discrepancy arises from

354 the fact that the colocalization approach only considers pairs of disease both associated 
medRxiv preprint doi: https://doi.org/10.1101/2022.01.25.22269831; this version posted January 28, 2022. The copyright holder for this preprint (which was not certified by peer review) is the author/funder, who has granted medRxiv a license to display the preprint in perpetuity.

It is made available under a CC-BY 4.0 International license .

355 at $P<5 \times 10^{-8}$ with genetic variants in overlapping credible sets. Our approach allows

356 associations not significant at $P<5 \times 10^{-8}$ to be retained (Extended Data Fig. 2).

\section{Discussion}

358 As genetic risk factors are becoming increasingly relevant to various fields of medicine,

359 the ability to evaluate their impact on disease burden is crucial. In this study we take

360 steps in this direction by presenting a new approach to quantify the effect of various

361 genetic exposures in terms of DALYs ("lost healthy life years") by combining genetic

362 data with DALY estimates from the Global Burden of Disease study. The results of our

363 main analyses can be freely explored at: https://dsge-lab.shinyapps.io/daly genetics/.

364 Overall, rare deleterious variants tended to have higher effects on DALYs than common

365 variants at the individual level. We also found that genetic exposures increasing the risk

366 of ischemic heart disease tended to be most impactful in terms of DALYs, since it

367 accounts for the largest share of population DALYs in the GBD for Finland 2019

368 (11.5\%) (ST3). Also, we have shown that the population level impact of some common

369 variants is comparable to important classic risk factors, such as low physical activity and

370 diet high in sodium. Polygenic scores, especially those that capture multiple diseases

371 (e.g., lifespan ${ }^{32}$ and multisite chronic pain $^{33}$ ), have a sizable impact both at individual

372 and population level (ST13).

373 The largest effects on individual DALYs were observed for carriers of high penetrance

374 deleterious rare variants in BRCA1 (breast and ovarian cancer), BRCA2 (breast,

375 ovarian, liver and prostate cancer), MYBPC3 (cardiomyopathy and myocarditis), LDLR

376 (ischemic heart disease) and MLH1 (colon and rectum cancer). However, due to the 
medRxiv preprint doi: https://doi.org/10.1101/2022.01.25.22269831; this version posted January 28, 2022. The copyright holder for this preprint (which was not certified by peer review) is the author/funder, who has granted medRxiv a license to display the preprint in perpetuity.

It is made available under a CC-BY 4.0 International license .

377 rarity of these variants, the population impact was at most 21 yearly population DALYs

378 per 100,000 for $B R C A 2$ (ST15), which is substantially lower than for the top common

379 variant rs7859727 (CDKN2B-CDKN2A) where minor allele carriers account for 447

380 yearly population DALYs per 100,000.

381 PGSes had a moderate to high impact both at the individual and population level. The

382 examined PGSes tended to moderately increase the risk of many diseases, with median

38316 out of 80 diseases retained per PGS. Overall, the top PGSes exert their effect

384 through cardiometabolic traits (e.g., through ischemic heart disease for shorter

385 lifespan ${ }^{32}$, coronary artery disease ${ }^{40}$ and type 2 diabetes ${ }^{41}$ PGSes) or pain/addiction-

386 related traits (e.g., through low back pain, substance use disorders, lung cancer and

387 COPD for multisite chronic pain ${ }^{33}$, lower educational attainment ${ }^{34}$, major depressive

388 disorder ${ }^{42}$, and smoking initiation ${ }^{43}$ PGSes). Note that the effect estimates for PGSes

389 depend on the cutoff used. For the shorter lifespan PGS, if we instead used top $1 \%$ vs

390 rest or top $50 \%$ vs rest cutoffs, the individual DALYs would have been 5.60 and 2.76

391 respectively, instead of the reported 3.81 for top $10 \%$ vs rest. We also note that the

392 effect of PGSes is in part a function of the predictive performance of each PGS, so their

393 relative importance can change and effect on DALYs will likely increase as larger

394 GWASes are used to construct them.

395 Most common variants with the highest effect on DALYs affected ischemic heart

396 disease risk (e.g., rs3798220, LPA; rs11591147, PCSK9; rs1537371, CDKN2B-

397 CDKN2A), and some affected risk of dementia (rs429358, APOE), prostate cancer

398 (rs183373024, POU5F1B), or type 2 diabetes (rs117361510, JPH2). The number of

399 DALYs for a disease in the GBD is driven by how common it is, how much premature 
400 death it causes (years of life lost, YLL), and how much and for how long it lowers quality

401 of life (years lived with disability, YLD). Common diseases that either lead to premature

402 mortality (high YLLs e.g., ischemic heart disease) and/or long periods of living with

403 disability (high YLDs, e.g., low back pain) account for a large number of population

404 DALYs (ST3). Consequently, genetic exposures increasing the risk of high-DALY

405 diseases predominated the results. Some variants might affect DALYs by modifying

406 intermediate risk factors such as BMI and blood pressure and, in our analyses, we have

407 included several variants that were associated with major risk factors. Nonetheless, we

408 noticed that most of the highest-ranking variants in terms of DALYs are associated

409 directly with the diseases rather than with intermediate modifiable risk factors

410 (rs8040868 in CHRNA5/A3/B4 being a notable exception due its impact on smoking

411 behavior).

412 There are multiple strengths and limitations to our study. One key strength is that the

413 genetic associations were estimated using individual-level data from two large biobank

414 studies with long registry-based follow-up. We apply a shrinkage procedure to the

415 associations, and thus obtain a conservative overview of the pleiotropic effect of genetic

416 exposures on 80 major disease accounting for $83.1 \%$ of the total DALYs in Finland

417 through all noncommunicable diseases in $2019^{19}$. In quantifying the disease burden we

418 combine the association results with population DALYs from the $\mathrm{GBD}^{19}$ which reports

419 perhaps most accurate and unbiased estimates of population-level disease burden. This

420 is important for many outcomes for which defining the absolute disease burden (e.g.,

421 incidence) through registry data is biased downwards. For example, quantifying the

422 number of individuals suffering from migraine using hospital episode statistics would 
medRxiv preprint doi: https://doi.org/10.1101/2022.01.25.22269831; this version posted January 28, 2022. The copyright holder for this preprint (which was not certified by peer review) is the author/funder, who has granted medRxiv a license to display the preprint in perpetuity.

It is made available under a CC-BY 4.0 International license .

423 lead to a serious underestimate (Extended Data Fig. 8). One important aspect of the

424 DALY estimates from the GBD is that the disease definitions are not overlapping and

425 the DALYs are comorbidity-corrected (see Supplementary Appendix Section 4.9 of GBD

$\left.4262019^{19}\right)$. Thus, double counting DALYs due to comorbidity is avoided. Finally, compared

427 to similar studies that consider modifiable/environmental risk factors, genetic exposures

428 are less likely to be impacted by confounding and other biases and thus more readily

429 allow for a causal interpretation.

430 In ST10 we provide an overview of the study limitations, some of which can be

431 overcome in future iterations of this work. Here we discuss perhaps the most important

432 ones. First, we take it as given that the DALYs estimated by the $\mathrm{GBD}^{19}$ are accurate

433 and that DALYs are a meaningful measure of disease burden (which has been

434 debated $^{44}$ ). Second, the DALYs for individuals with a disease is assumed to be the

435 same among those with and without the genetic exposures (e.g., individuals with and

436 without a damaging BRCA1 mutation that develop breast cancer accumulate DALYs

437 similarly). Third, we used DALYs estimated for Finland 2019 and estimated genetic

438 associations using data between 1972 and 2020 in Finland and United Kingdom, so the

439 estimated effects on lifetime individual DALYs for someone born today can change as

440 the disease incidence, medical care, and mediating factors (e.g., smoking) change.

441 Also, we currently do not model gene-environment effects or account for age-varying

442 effects. Fourth, although we suggest a causal interpretation to the attributable DALYs,

443 there are important caveats to be made. Despite rigorous statistical fine-mapping, the

444 reported variants with the highest posterior probability might tag underlying causal

445 genetic variation. For example, the rs3798220 (LPA) variant is known to tag copy 
446 number variation of the Kringle IV like domain 2 in the LPA locus, which is the probable

447 mechanism behind the association of rs3798220 with ischemic heart disease ${ }^{45}$. Another

448 caveat lies in the possibility that a reported variant does not tag one causal variant, but

449 multiple causal variants in LD. However, using eQTL data, previous work on quantitative

450 lipid traits has shown that a minority of pleiotropic effects at a given locus are explained

451 by this configuration ${ }^{46}$. Finally, note that we mainly present attributable DALYs for both

452 sexes in aggregate, which might be misleading for exposures with sex-specific effects.

453 For example, rs183373024 (POU5F1B) affected DALYs only through prostate cancer

454 and benign prostatic hyperplasia (0.54 DALYs in aggregate, 1.06 DALYs in males, 455 ST11).

456 The presented framework can be applied to other countries and ancestries under 457 certain assumptions. First, the effect sizes of the genetic exposures need to be 458 generalizable to the target population. There is increasing evidence that many causal 459 variants have similar effects across continental ancestry groups ${ }^{47-51}$, but this does not 460 apply to polygenic scores ${ }^{52}$. Assuming effect sizes are consistent, one needs to know 461 the frequency of the genetic exposures in the target population, which is particularly 462 challenging in countries with a heterogeneous ancestry composition. In the absence of 463 representative genetic surveys, it might be possible to use self-reported ancestry 464 information combined with frequency datasets such as gnomAD ${ }^{53}$.

465 In conclusion, we have proposed an approach to combine genetic association results 466 from large biobank studies with disease burden estimates from the GBD and provided 467 an overview of the impact of genetic exposures on lost healthy life years (DALYs). We 468 have shown that some common variants account for as many healthy life years as 
469 some well-established modifiable risk factors. We emphasize that beyond estimating the

470 direct impact of genetic exposures on DALYs, genetic results can be used to better

471 estimate the causal effect of modifiable risk factors on disease burden using techniques

472 such as Mendelian randomization ${ }^{54}$. While genetic risk factors are not yet modifiable in

473 practice, novel approaches based on in vivo gene editing are being developed ${ }^{16}$ and

474 some have been tested in clinical trials ${ }^{17,18}$. Estimating the total impact of genetic risk

475 factors on disease burden might help prioritize these types of interventions. 


\section{References}

477 1. Visscher, P. M. et al. 10 Years of GWAS Discovery: Biology, Function, and 478 Translation. Am J Hum Genet 101, 5-22 (2017).

479 2. Watanabe, K. et al. A global overview of pleiotropy and genetic architecture in $480 \quad$ complex traits. Nat Genet 51, 1339-1348 (2019).

3. Mars, N. et al. The role of polygenic risk and susceptibility genes in breast cancer over the course of life. Nat Commun 11, 6383 (2020).

4. Rasmussen, K. L., Tybjærg-Hansen, A., Nordestgaard, B. G. \& Frikke-Schmidt, R. Absolute 10-year risk of dementia by age, sex and APOE genotype: a populationbased cohort study. CMAJ 190, E1033-E1041 (2018).

5. Metcalfe, K. A. et al. The risk of breast cancer in BRCA1 and BRCA2 mutation carriers without a first-degree relative with breast cancer. Clin Genet 93, 1063-1068 (2018).

6. Li, T. et al. Total genetic contribution assessment across the human genome. Nat Commun 12, 2845 (2021).

7. Mars, N. et al. Polygenic and clinical risk scores and their impact on age at onset and prediction of cardiometabolic diseases and common cancers. Nat Med 26, 549557 (2020).

8. Sakaue, S. et al. Trans-biobank analysis with 676,000 individuals elucidates the association of polygenic risk scores of complex traits with human lifespan. Nature Medicine 26, 542-548 (2020).

9. Murray, C. J. L. et al. Global burden of 87 risk factors in 204 countries and territories, 1990-2019: a systematic analysis for the Global Burden of Disease Study 2019. The Lancet 396, 1223-1249 (2020).

10. Sun, L. et al. Polygenic risk scores in cardiovascular risk prediction: A cohort study and modelling analyses. PLoS Med 18, e1003498 (2021). 
502 11. Lewis, C. M. \& Vassos, E. Polygenic risk scores: from research tools to clinical 503 instruments. Genome Med 12, 44 (2020).

504 12. Manchanda, R. \& Gaba, F. Population Based Testing for Primary Prevention: A 505 Systematic Review. Cancers (Basel) 10, E424 (2018).

506 13. Turley, P. et al. Problems with Using Polygenic Scores to Select Embryos. N Engl J 507 Med 385, 78-86 (2021).

508 14. Lencz, T. et al. Utility of polygenic embryo screening for disease depends on the 509 selection strategy. bioRxiv 2020.11.05.370478 doi:10.1101/2020.11.05.370478.

511 15. Karavani, E. et al. Screening Human Embryos for Polygenic Traits Has Limited $512 \quad$ Utility. Cell 179, 1424-1435.e8 (2019).

513 16. Musunuru, K. et al. In vivo CRISPR base editing of PCSK9 durably lowers 514 cholesterol in primates. Nature 593, 429-434 (2021).

515 17. Frangoul, $H$. et al. CRISPR-Cas9 Gene Editing for Sickle Cell Disease and $\beta$ $516 \quad$ Thalassemia. N Engl J Med 384, 252-260 (2021).

517 18. Rim, J. H., Gopalappa, R. \& Gee, H. Y. CRISPR-Cas9 In Vivo Gene Editing for $518 \quad$ Transthyretin Amyloidosis. N Engl J Med 385, 1722 (2021).

519 19. Vos, T. et al. Global burden of 369 diseases and injuries in 204 countries and 520 territories, 1990-2019: a systematic analysis for the Global Burden of Disease Study 2019. The Lancet 396, 1204-1222 (2020).

522 20. Wang, G., Sarkar, A., Carbonetto, P. \& Stephens, M. A simple new approach to 523 variable selection in regression, with application to genetic fine mapping. Journal of the Royal Statistical Society: Series B (Statistical Methodology) 82, 1273-1300 525 (2020).

526 21. Kanai, M. et al. Insights from complex trait fine-mapping across diverse populations. 2021.09.03.21262975

528 https://www.medrxiv.org/content/10.1101/2021.09.03.21262975v1 doi:10.1101/2021.09.03.21262975. 
530 22. Ritari, J. et al. Increasing accuracy of HLA imputation by a population-specific reference panel in a FinnGen biobank cohort. NAR Genomics and Bioinformatics 2, (2020).

$534 \quad$ European ancestry. Nature communications 9, (2018).

535 24. Schlaepfer, I. R. et al. The CHRNA5/A3/B4 gene cluster variability as an important determinant of early alcohol and tobacco initiation in young adults. Biol Psychiatry

538 25. Joshi, P. K. et al. Variants near CHRNA3/5 and APOE have age- and sex-related effects on human lifespan. Nat Commun 7, 11174 (2016).

540 26. Green, R. C. et al. ACMG recommendations for reporting of incidental findings in clinical exome and genome sequencing. Genet Med 15, 565-574 (2013).

27. Miller, D. T. et al. ACMG SF v3.0 list for reporting of secondary findings in clinical exome and genome sequencing: a policy statement of the American College of

545 28. Landrum, M. J. et al. ClinVar: public archive of relationships among sequence 546 variation and human phenotype. Nucleic Acids Res 42, D980-985 (2014).

547 29. Spurdle, A. B. et al. ENIGMA--evidence-based network for the interpretation of 548 germline mutant alleles: an international initiative to evaluate risk and clinical significance associated with sequence variation in BRCA1 and BRCA2 genes. Hum Mutat 33, 2-7 (2012).

551 30. McLaren, W. et al. The Ensembl Variant Effect Predictor. Genome Biol 17, 122 552 (2016).

553 31. Liu, J. Z. et al. Association analyses identify 38 susceptibility loci for inflammatory 554 bowel disease and highlight shared genetic risk across populations. Nat Genet 47, 979-986 (2015). 
556 32. Timmers, P. R. et al. Genomics of 1 million parent lifespans implicates novel 557 pathways and common diseases and distinguishes survival chances. Elife 8, 558 e39856 (2019).

559 33. Johnston, K. J. A. et al. Genome-wide association study of multisite chronic pain in $560 \quad$ UK Biobank. PLoS Genet 15, (2019).

561 34. Lee, J. J. et al. Gene discovery and polygenic prediction from a genome-wide 562 association study of educational attainment in 1.1 million individuals. Nat Genet 50, 563 1112-1121 (2018).

564 35. BasuRay, S. PNPLA3-I148M: a problem of plenty in non-alcoholic fatty liver disease. $565 \quad$ Adipocyte 8, 201-208 (2019).

566 36. Chheda, $\mathrm{H}$. et al. Whole-genome view of the consequences of a population bottleneck using 2926 genome sequences from Finland and United Kingdom. Eur J Hum Genet 25, 477-484 (2017).

569 37. Lim, E. T. et al. Distribution and medical impact of loss-of-function variants in the Finnish founder population. PLoS Genet 10, e1004494 (2014).

571 38. Ruotsalainen, S. E. et al. Loss-of-function of MFGE8 and protection against coronary atherosclerosis. 2021.06.23.21259381

573 https://www.medrxiv.org/content/10.1101/2021.06.23.21259381v1 doi:10.1101/2021.06.23.21259381.

39. Stensrud, M. J. \& Hernán, M. A. Why Test for Proportional Hazards? JAMA 323, 1401-1402 (2020).

40. Nikpay, M. et al. A comprehensive 1,000 Genomes-based genome-wide association

41. Mahajan, A. et al. Fine-mapping type 2 diabetes loci to single-variant resolution 580 using high-density imputation and islet-specific epigenome maps. Nat Genet 50, 1505-1513 (2018). 
medRxiv preprint doi: https://doi.org/10.1101/2022.01.25.22269831; this version posted January 28, 2022. The copyright holder for this preprint (which was not certified by peer review) is the author/funder, who has granted medRxiv a license to display the preprint in perpetuity.

It is made available under a CC-BY 4.0 International license .

582 42. Wray, N. R. et al. Genome-wide association analyses identify 44 risk variants and 583 refine the genetic architecture of major depression. Nature Genetics 50, 668-681 584 (2018).

585 43. Liu, M. et al. Association studies of up to 1.2 million individuals yield new insights 586 into the genetic etiology of tobacco and alcohol use. Nature Genetics 51, 237-244 587 (2019).

44. Shiffman, J. \& Shawar, Y. R. Strengthening accountability of the global health metrics enterprise. Lancet 395, 1452-1456 (2020).

590

45. Mukamel, R. E. et al. Protein-coding repeat polymorphisms strongly shape diverse human phenotypes. Science 373, 1499-1505 (2021).

592

46. Giambartolomei, C. et al. Bayesian test for colocalisation between pairs of genetic association studies using summary statistics. PLoS Genet 10, e1004383 (2014).

594 47. Guo, J. et al. Quantifying genetic heterogeneity between continental populations for human height and body mass index. Sci Rep 11, 5240 (2021).

596 48. Kuchenbaecker, K. et al. The transferability of lipid loci across African, Asian and European cohorts. Nat Commun 10, 4330 (2019).

598 49. Huang, Q. Q. et al. Transferability of genetic loci and polygenic scores for cardiometabolic traits in British Pakistanis and Bangladeshis. 2021.06.22.21259323 https://www.medrxiv.org/content/10.1101/2021.06.22.21259323v1 doi:10.1101/2021.06.22.21259323.

602 50. Shi, H. et al. Population-specific causal disease effect sizes in functionally important 603 regions impacted by selection. Nat Commun 12, 1-15 (2021).

604 51. Patel, R. A. et al. Effect sizes of causal variants for gene expression and complex 605 traits differ between populations. 2021.12.06.471235

606 https://www.biorxiv.org/content/10.1101/2021.12.06.471235v1

607 doi:10.1101/2021.12.06.471235.

608 52. Martin, A. R. et al. Clinical use of current polygenic risk scores may exacerbate 609 health disparities. Nat Genet 51, 584-591 (2019). 
medRxiv preprint doi: https://doi.org/10.1101/2022.01.25.22269831; this version posted January 28, 2022. The copyright holder for this preprint (which was not certified by peer review) is the author/funder, who has granted medRxiv a license to display the preprint in perpetuity. It is made available under a CC-BY 4.0 International license.

610 53. Karczewski, K. J. et al. The mutational constraint spectrum quantified from variation 611 in 141,456 humans. Nature 581, 434-443 (2020).

612 54. Davey Smith, G. \& Ebrahim, S. 'Mendelian randomization': can genetic 613 epidemiology contribute to understanding environmental determinants of disease? $614 \quad$ Int J Epidemiol 32, 1-22 (2003).

615 


\section{Methods}

\section{General statistical methods}

618 All analyses were performed using $\mathrm{R}$ version 4.0.3. All $P$-values are nominal. Error bars

619 represent $95 \%$ confidence intervals. Confidence intervals for the disease-specific

620 DALYs were generated using the delta method. Confidence intervals for total DALYs for

621 each exposure were estimated by repeating 10,000 times the computation of total

622 DALYs while resampling the log-HR estimates from a multivariate normal distribution

623 corresponding to the approximate sampling distribution of log-HRs, and by taking the

$6242.5^{\text {th }}$ and $97.5^{\text {th }}$ percentiles as the bounds for the $95 \%$ confidence intervals

625 (Supplementary Information). The sex-specific results were generated through

626 estimating the HRs stratified by sex and using sex-specific population DALYs reported

627 by the $\mathrm{GBD}^{19}$. For all error estimates we assumed there was no uncertainty in

628 frequencies of the exposures (e.g., allele frequencies) or DALYs reported by the GBD,

629 so we only consider variation from HR estimation in the error estimates.

\section{Participants}

631 FinnGen and UK Biobank ${ }^{55}$ are large-scale population-based prospective cohort studies

632 from Finland and the United Kingdom, both with phenotypic data ascertained from

633 registries (Fig. 1). In FinnGen, we used participants from the Finnish ancestry in data

634 freeze 7 with $n=309,136$ (56.2\% female) with median (IQR) age at start of follow-up of

$63515.1(0-26.3)$ and at end of follow-up 62.2 (47.1-72.9). The median (IQR) follow-up

636 length was $47.3(41.6-47.7)$ years (ST1). Follow-up for FinnGen started on January $1^{\text {st }}$,

6371972 and ended on August $31^{\text {st }}, 2020$. For UKB we used participants of European

638 ancestry $n=426,612$ ( $54.1 \%$ female) with median (IQR) age at start of follow-up of 47.2 
medRxiv preprint doi: https://doi.org/10.1101/2022.01.25.22269831; this version posted January 28, 2022. The copyright holder for this preprint (which was not certified by peer review) is the author/funder, who has granted medRxiv a license to display the preprint in perpetuity.

It is made available under a CC-BY 4.0 International license .

639 (39.5-52.4) and at end of follow-up 69.1 (61.7-74.2). The median (IQR) follow-up length

640 was $22.3(22.3-22.3)$ years (ST1). Follow-up for UKB started on January $1^{\text {st }}, 1998$ and

641 ended on April 30th, 2020.

\section{Disability-adjusted life years}

643 As a measure of disease burden we used the disability-adjusted life years (DALYs) from

644 the 2019 Global Burden of Disease study (GBD) ${ }^{19}$ with data publicly available at

645 http://ghdx.healthdata.org/. DALYs are a metric for measuring population-level disease

646 burden that combines a measure of premature mortality called years of life lost (YLL)

647 and a measure of healthy life years lost due to lowered quality of life called years lived

648 with disability (YLD), so DALYs are the sum of YLDs and YLLs (Extended Data Fig. 1).

649 The GBD is a longitudinal study which estimates incidence, prevalence, mortality, YLLs,

650 YLDs and DALYs due to various collectively exhaustive and mutually exclusive

651 diseases and injuries (369 in 2019) for hundreds of countries (204 in 2019) ${ }^{19}$. GBD

652 strives to model unbiased estimates using various sources of information, including

653 census data, household surveys, civil registration and vital statistics, disease registries,

654 health service use data, and more. The estimation process for DALYs is complex and

655 varies from disease to disease, see the 2019 GBD publication ${ }^{19}$ and its Supplementary

656 Appendix 1 for a detailed description of the methods. Because all of our genetic 657 exposures, except rare variants, were measured in FinnGen, we used the 2019 GBD

658 metrics for Finland and present all estimates for Finland, although the estimates could 659 be calculated for any country in the GBD given some assumptions. See ST3 for a list of 660 DALYs, YLLs and YLDs for the included diseases. 
medRxiv preprint doi: https://doi.org/10.1101/2022.01.25.22269831; this version posted January 28, 2022. The copyright holder for this preprint (which was not certified by peer review) is the author/funder, who has granted medRxiv a license to display the preprint in perpetuity.

It is made available under a CC-BY 4.0 International license .

\section{Disease phenotypes}

663 To define the disease phenotypes, we manually mapped 89 mutually exclusive

664 noncommunicable diseases from the 2019 GBD to FinnGen clinical endpoints (ST2), of

665 which we retained 80 based on requiring at least 500 individuals to have the disease in

666 FinnGen. These 80 diseases account for $83.1 \%$ of population DALYs from

667 noncommunicable diseases in Finland 2019 (ST3). We did not map diseases from the

668 GBD that were too rare, not well enough captured by the registries, or include too

669 heterogenous a set of diseases (e.g., "other cardiovascular and circulatory diseases").

670 Phenotyping in FinnGen relied on information on diagnoses starting from 1972 in the

671 hospital discharge registry ${ }^{56}$, the causes of death registry, and the cancer registry. The

672 drug purchases registry was additionally used starting from 1995 for selected diseases

673 (e.g., migraine). We completely excluded diseases with less than 500 cases in FinnGen.

674 Phenotyping in UKB was performed via groups of ICD-10 codes mapped from the

675 FinnGen endpoints (ST2), relying on ICD-10 diagnosis codes from the hospital episode

676 statistics, cancer registry, and causes of death registry data starting from 1998. The

677 cumulative incidence of the diseases varied from $14.93 \%$ (cataract) to $0.16 \%$ (acute

678 glomerulonephritis) in FinnGen. We analyzed the following diseases only in FinnGen as

679 in UKB there were less than 500 cases: Hemoglobinopathies and hemolytic anemias

$680(n=480$ in UKB), Hodgkin Lymphoma $(n=464)$, autism spectrum disorders $(n=164)$,

681 eating disorders $(n=163)$, acne vulgaris $(n=106)$, acute glomerulonephritis $(n=58)$,

682 attention-deficit hyperactivity disorder $(n=26)$, and conduct disorder $(n=13)$. As both

683 FinnGen and UKB rely mainly on diagnosis codes recorded at hospitals, conditions that 
medRxiv preprint doi: https://doi.org/10.1101/2022.01.25.22269831; this version posted January 28, 2022. The copyright holder for this preprint (which was not certified by peer review) is the author/funder, who has granted medRxiv a license to display the preprint in perpetuity.

It is made available under a CC-BY 4.0 International license .

684 are usually managed in the primary or outpatient care setting are under-ascertained, for 685 examples see Extended Data Fig. 8.

\section{Common variants}

687 We provide information on genotyping, imputation, quality control, fine-mapping, and LD 688 clumping in Supplementary Information. For each variant, we defined the minor allele

689 to be the allele less common in FinnGen. Due to symmetry in attributable DALY 690 estimation, going from 1 to 0 copies vs 0 to 1 copies of an allele only changes the sign

691 of the individual attributable DALYs estimates. After fine-mapping, LD clumping, and 692 shrinkage, we included a total 1044 common variants (FinnGen MAF>0.01) for the 693 analysis (Fig. 1). FinnGen SuSiE fine-mapping ${ }^{20}$ results for all FinnGen endpoints used

694 to define the 80 diseases (ST2) were used to select, for each credible set, the genome695 wide significant variant $\left(\mathrm{P}<5 \times 10^{-8}\right)$ with the highest posterior inclusion probability, 696 resulting in 564 variants. Additionally, we included all FinnGen coding variants with a $697 \mathrm{P}<5 \times 10^{-8}$ association with any FinnGen endpoint $(n=325)$. We used UKB fine-mapping 698 results $^{21}$ for continuous risk factor traits (BMI, systolic blood pressure, cigarettes per 699 day, HLD-C, LDL-C, and HbA1c) and selected, for each credible set, variants with $700 \mathrm{P}<5 \times 10^{-12}$ and the highest posterior inclusion probability, which resulted in inclusion of

701155 additional variants. We labeled common variants as Finnish-enriched if they had 702 FinnGen MAF enrichment at least 5-fold compared to the non-Finnish-non-Swedish703 non-Estonian European (NFSEE) ancestry MAF and the NFSEE MAF was over 0.01. In

704 ST5 we report all common variants included in the analysis. We separately imputed ${ }^{22} 74$

705 alleles in 7 HLA genes (ST9, Supplementary Information) for the HLA region 
medRxiv preprint doi: https://doi.org/10.1101/2022.01.25.22269831; this version posted January 28, 2022. The copyright holder for this preprint (which was not certified by peer review) is the author/funder, who has granted medRxiv a license to display the preprint in perpetuity.

It is made available under a CC-BY 4.0 International license .

706 (chr6:29691116 to chr6:3054976 in GRCh38) and determined 6 haplotypes (ST8) for

707 APOE.

\section{Rare deleterious variants}

709 To analyze the effects of rare deleterious variants on DALYs, we used whole exome-

710 sequencing data from a subset of participants in UKB $(n=174,379)$ from the December

7112020 release. We processed the data using Hail version 0.2.59-63cf625e29e5. We

712 directly processed the quality controlled $P L I N K^{57}$ files provided by UKB. We annotated

713 the data using the Ensembl Variant Effect Predictor ${ }^{30}$ using the same approach used in

714 gnomAD and specifically the function gnomadvep.vep_or_lookup_vep. VEP annotations

715 were processed using the function gnomadvep.process_consequences, consistently

716 with the gnomAD definition of pLOF, missense and synonymous variants, using the

717 canonical transcript. We also extracted $\mathrm{ClinVar}^{28}$ annotated variants (accessed in

718 November 2020) and germline variants in BRCA1 and BRCA2 (accessed in November

719 2020) annotated by the ENIGMA consortium ${ }^{29}$. From ClinVar we considered

720 "pathogenic" and "likely-pathogenic" variants (no filtering on star status or number of

721 submitters) and from ENIGMA we considered "pathogenic" variants. Variants with

722 frequency greater than 0.001 were excluded because they are less likely to be

723 deleterious. We considered all genes part of the the American College of Medical

724 Genetics and Genomics (ACMG) recommendations for reporting of incidental findings in

725 clinical exome and genome sequencing studies ${ }^{27}$. We formed two types of rare variant

726 burdens for individuals for each gene: 1) the pLOF burden was set as positive if there

727 was at least 1 variant annotated as pLOF and 2) the ClinVar/ENIGMA burden was set

728 as positive if there was at least 1 variant annotated as "pathogenic" in ENIGMA ${ }^{29}$ for 
medRxiv preprint doi: https://doi.org/10.1101/2022.01.25.22269831; this version posted January 28, 2022. The copyright holder for this preprint (which was not certified by peer review) is the author/funder, who has granted medRxiv a license to display the preprint in perpetuity.

It is made available under a CC-BY 4.0 International license .

$729 B R C A 1$ and BRCA2 and for other genes "likely pathogenic" or "pathogenic" in ClinVar.

730 Due to statistical power considerations, we restricted our analysis so that at least 35

731 individuals had a positive burden, resulting in 9 genes for both burden types (ST4).

\section{Polygenic scores}

733 In FinnGen, we included 30 genome-wide polygenic scores (PGS) for traits of interest

734 constructed from publicly available summary statistics, see ST6 for references. We

735 selected PGSes for psychobehavioral traits (e.g. cognitive ability, neuroticism), major

736 chronic diseases (e.g. coronary artery disease, depression), and major risk factors (e.g.

737 LDL-C, blood pressure) to cover traits of interest with high quality summary statistics

738 available. PGSes were only analyzed in FinnGen, since many of the original summary

739 statistics included UK Biobank. We used PRS-CS ${ }^{58}$ for generating the PGSes using

740 external LD reference panel (1000 Genomes Europeans). We used the PRS-CS-auto

741 algorithm, which learns the model's global scaling parameter $\square$ from the data and

742 performs well with large datasets. Default PRS-CS parameters were used and only

743 HapMap 3 SNPs were considered (see https://github.com/FINNGEN/CS-PRS-pipeline

744 for code). Scores for lifespan, educational attainment, cognitive performance, and

745 intelligence were reversed before analysis to make all scores on net deleterious in

746 terms of DALYs. For defining the exposure for survival models, we coded individuals for

747 each PGS as 1 if they were in the top $10 \%$ of the score distribution and 0 if not.

748 Consequently, the individual DALYs can be interpreted as the effect on lifetime DALYs if

749 the average individual in top $10 \%$ of the PGS were to have their PGS be that of the

750 average in the bottom $90 \%$ of the PS. 


\section{Survival models}

753 To estimate the HRs between all genetic exposure-disease pairs we used Cox

754 proportional hazards regression via the coxph function in the survival package version

755 3.2-11. The model was additive for allele counts. Sex and first 10 principal components

756 of population structure were included as covariates. We used calendar age as the time-

757 scale and age at first record of the disease in the registries as time-to-event. Individuals

758 were censored at death, emigration, or end of registry-based follow-up (August $31^{\text {st }}$

7592020 in FinnGen, April $30^{\text {th }} 2020$ in UKB). For the common variants, the HRs were

760 estimated both in FinnGen and UKBB separately and combined using fixed effects

761 inverse-variance weighted meta-analysis. A comparison of effect sizes between

762 FinnGen and UK biobank is provided in Extended Data Fig. 3. We did not account for

763 left censoring, nor did we account for relatedness of the subjects due to computational

764 limitations. For the rare variant burden analysis in UKB, we used Cox regression with

765 Firth's Penalized Likelihood ${ }^{59}$ via coxphf package version 1.13.1 and included sex and

766 the 4 first genetic principal components as covariates.

767 For the sensitivity analysis in FinnGen accounting for relatedness in estimating standard

768 errors for the log-HRs, we generated a family indicator from genotype data using

$769 \mathrm{KING}^{60}$ version 2.2 only including HapMap 3 variants. Individuals up to $3^{\text {rd }}$ degree of

770 relatedness were included in the same family. The robust standard errors were

771 estimated using a family indicator to compute robust standard errors by including

772 cluster(family_id) as a covariate in coxph.

773 


\section{Shrinkage}

775 We use prior information to apply a shrinkage procedure to the HRs for exposure-

776 disease pairs to reduce the effect of sampling variation on our results. Denote by $b_{e, d}$

777 the log-HR of genetic exposure $e$ on disease $d=1, . ., 80$. One genetic exposure at a time,

778 we set the prior distribution of $b_{e, d}$, to be a mixture distribution between the point mass

779 at 0 and a 50:50 mixture of two normal distributions with means at -0.3 and 0.3 ,

780 respectively, and with a standard deviation of 0.1 . We denote the mixture weight of the

781 non-zero component by $p_{e}$, which can be interpreted as the exposure-specific proportion

782 of non-zero effects across the 80 diseases. We set the prior distribution of $p_{e}$ to a

$783 \operatorname{Beta}(\alpha=1, \beta=19)$ distribution, that has an expected value of 0.05 . The full probability

784 model is

$$
\begin{gathered}
p_{e} \sim \operatorname{Beta}(\alpha, \beta), \pi_{e, d} \sim \operatorname{Bernoulli}(0.5), \\
b_{e, d} \sim \operatorname{Bernoulli}\left(p_{e}\right)\left[\left(1-\pi_{e, d}\right) \mathcal{N}\left(\mu, \sigma^{2}\right)+\pi_{e, d} \mathcal{N}\left(-\mu, \sigma^{2}\right)\right], \\
\text { where } \alpha=1, \beta=19, \mu=0.3 \text { and } \sigma=0.1 .
\end{gathered}
$$

785 This model implies that, before seeing the data, for each genetic exposure, we expect a

786 non-zero effect for $4(=0.05 \times 80)$ diseases and the direction of the non-zero effects are

787 equally likely to be risk increasing (centered around $H R=1.35$ ) as protective (centered

788 around $H R=0.74)$. In practice, which effects are shrunk to zero and which are retained

789 as non-zero, does not vary considerably when these prior parameters are varied

790 (Extended Data Fig. 6). For each genetic exposure $e$ at a time, we use a Markov Chain

791 Monte Carlo procedure with 10,000 iterations (Supplementary Code) to estimate the

792 posterior probabilities of the log-HRs $\left(b_{e, d}\right)$ for diseases $d=1,2, \ldots, 80$ coming from the 
793 null model (point mass at zero). We discard any log-HRs where the null probability is

794 over 10\%, and, for the remaining log-HRs, we use the maximum partial likelihood

795 estimates from the Cox proportional hazards model in the downstream analyses.

\section{Attributable disability-adjusted life years}

797 Similarly to the $\mathrm{GBD}^{9}$, we use the HRs and frequencies of the exposures to estimate

798 attributable DALYs one disease at a time. We use the multilevel exposure formula ${ }^{61}$ for

799 the population attributable fraction (i.e., the fraction of cases of disease $d$ caused by the

800 exposure levels in the population deviating from counterfactual levels):

$$
\text { Population attributable fraction }=\mathrm{AFp}_{\mathrm{d}}=\frac{\sum_{i} \mathrm{P}_{i} \mathrm{HR}_{d, i}-\sum_{i} \mathrm{P}_{i}^{\prime} \mathrm{HR}_{d, i}}{\sum_{i} \mathrm{P}_{i} \mathrm{HR}_{d, i}}
$$

801 where $\mathrm{HR}_{d, i}$ is the hazard ratio for disease $d$ at exposure level $i$ (e.g., 1 copy) relative to

802 reference (e.g., 0 copies) and $\mathrm{P}_{i}$ is the fraction of the population at exposure level $i$ and

$803 \mathrm{P}_{i}^{\prime}$ represents the fraction of the population at exposure level $i$ in the counterfactual

804 scenario (e.g., if all with 1 copy instead had $0: \mathrm{P}_{1}^{\prime}=0, \mathrm{P}_{0}^{\prime}=\mathrm{P}_{0}+\mathrm{P}_{1}$, and $\mathrm{P}_{2}^{\prime}=\mathrm{P}_{2}$ ).

805 As an example, for individuals carrying 0,1 , and 2 alleles with respective population

806 frequencies of $\mathrm{P}_{0}=0.7, \mathrm{P}_{1}=0.2$, and $\mathrm{P}_{2}=0.1$, and $\mathrm{HRs}$ for disease $d$ of $\mathrm{HR}_{d, 0}=1.00$,

$807 \mathrm{HR}_{d, 1}=1.35$, and $\mathrm{HR}_{d, 2}=1.82$. For calculating attributable DALYs from individuals

808 carrying 1 vs 0 copies of the allele, define the counterfactual frequencies as $\mathrm{P}_{0}^{\prime}=0.9$,

$809 \mathrm{P}_{1}^{\prime}=0.0$, and $\mathrm{P}_{2}^{\prime}=0.1$ (i.e., making all with 1 copy have 0 copies instead). Plugging

810 these numbers into the $\mathrm{AFp}_{\mathrm{d}}$ formula produces the population attributable fraction of

811 disease cases from those carrying 1 vs 0 copies of the allele (the fraction of cases that

812 would be prevented if all with 1 allele had instead 0 ), which is 0.061 in this case, so 
813 approximately $6.1 \%$ of disease cases would be prevented if all with 1 copy had instead

8140 copies at birth.

815 We then assume that the estimated population attributable fraction of disease cases

816 can be interpreted as the population attributable fraction of DALYs, which is true if all

817 disease cases contribute on average the same amount of DALYs independent of

818 whether they have the genetic exposure or not. This assumption does not hold if, for

819 example, deleterious BRCA1 mutation carriers develop breast cancer earlier and

820 consequently accumulate more DALYs per case. Then, multiplying the population

821 attributable fraction of DALYs $\left(\mathrm{AFp}_{\mathrm{d}}\right)$ by the population DALYs per year per 100,000

822 reported by the GBD $\left(\mathrm{DALY}_{d}\right)$ gives the population attributable DALYs, interpreted in our

823 example as the expected loss of healthy life years per year per 100,000 if the population

824 frequencies of the exposure were $\mathrm{P}_{i}^{\prime}$ instead of $\mathrm{P}_{i}$ (in our example, all with 1 copy had

825 instead 0 copies).

Population attributable DALYs ${ }_{\mathrm{d}}=\mathrm{AFp}_{\mathrm{d}} \times \mathrm{DALY}_{d}$

826 We further estimate individual attributable $D A L Y S$ for binary counterfactuals (e.g., 827 having 1 vs 0 copies, being in top $10 \%$ of a PS vs bottom $90 \%$ ) by dividing the 828 population attributable DALYs per 100,000 by the number of individuals with the 829 exposure out of $100,000\left(100,000 \times P_{i}\right)$ and multiplying by life-expectancy at birth $(\mathrm{L})$ :

Individual attributable DALYs $s_{\mathrm{d}}=\frac{\mathrm{AFp}_{\mathrm{d}} \times \mathrm{DALY}_{d}}{100,000 \times \mathrm{P}_{i}}=\frac{\text { Population attributable DALYs }_{\mathrm{d}}}{\text { No. individuals exposed per 100,000 }}$

830 where $\mathrm{DALY}_{d}$ represents the population DALYs per year per 100,000 through disease $d$ 831 from GBD, $P_{i}$ is the fraction of population for which the exposure is changed (e.g.,

832 fraction of those with 1 copy), and L represents the life-expectancy at birth (which is 43 
833 included to convert yearly DALYs into lifetime estimates). These individual DALYs are

834 interpreted as the expected loss of healthy life years for an individual caused by having

835 the genetic exposure at birth. Finally, both population attributable DALYs and individual

836 DALYs can be summed up across the 80 diseases to arrive at the total impact of the

837 genetic exposure. See Witte et al. ${ }^{62}$ for discussion on how population attributable

838 fractions relate to other measures of genetic contribution.

839 References

840 55. Sudlow, C. et al. UK Biobank: An Open Access Resource for Identifying the Causes 841 of a Wide Range of Complex Diseases of Middle and Old Age. PLOS Medicine 12, 842 e1001779 (2015).

843 56. Sund, R. Quality of the Finnish Hospital Discharge Register: A systematic review.

844 Scandinavian Journal of Public Health 40, 505-515 (2012).

845 57. Purcell, S. et al. PLINK: A Tool Set for Whole-Genome Association and Population846 Based Linkage Analyses. Am J Hum Genet 81, 559-575 (2007).

847 58. Ge, T., Chen, C.-Y., Ni, Y., Feng, Y.-C. A. \& Smoller, J. W. Polygenic prediction via 848 Bayesian regression and continuous shrinkage priors. Nature Communications 10, 1776 (2019).S

850 59. Heinze, G. \& Schemper, M. A solution to the problem of monotone likelihood in Cox 851 regression. Biometrics 57, 114-119 (2001).

852 60. Manichaikul, A. et al. Robust relationship inference in genome-wide association 853 studies. Bioinformatics 26, 2867-2873 (2010).

854 61. Khosravi, A., Nazemipour, M., Shinozaki, T. \& Mansournia, M. A. Population 855 attributable fraction in textbooks: Time to revise. Global Epidemiology 3, 100062 856 (2021).

857 62. Witte, J. S., Visscher, P. M. \& Wray, N. R. The contribution of genetic variants to 858 disease depends on the ruler. Nat Rev Genet 15, 765-776 (2014). 
medRxiv preprint doi: https://doi.org/10.1101/2022.01.25.22269831; this version posted January 28, 2022. The copyright holder for this preprint (which was not certified by peer review) is the author/funder, who has granted medRxiv a license to display the preprint in perpetuity.

859 


\section{Data availability}

861 We present all attributable DALY results in ST8, ST9, and ST11-ST16. Results for

862 common variants, HLA alleles, PGSes can be explored through plots at https://dsge-

863 lab.shinyapps.io/daly genetics/

864 Code for central parts of analyses can be found at

865 https://github.com/dsgelab/dalys code

\section{Acknowledgements}

867 We thank the whole FinnGen team for their contribution. In particular, we thank Mari K.

868 Niemi for methodological support and comments to the manuscript, Samuli Ripatti and

869 Masahiro Kanai for insightful comments on the manuscript, Pietro della Briotta Parolo

870 for constructing the polygenic scores and kinship information in FinnGen. We also thank

871 John J. McGrath (Aarhus University) for very helpful comments on the manuscript. S.J.

872 was supported by the Academy of Finland (grant no. 341747). A.G. was supported by

873 Academy of Finland (grant no. 323116) and by the European Research Council (ERC)

874 under the European Union's Horizon 2020 research and innovation programme (grant

875 no. 945733). This project has also received funding from the European Union's Horizon

8762020 research and innovation programme under grant agreement no. 101016775.

877 The FinnGen project is funded by two grants from Business Finland (HUS 4685/31/2016

878 and UH 4386/31/2016) and nine industry partners (AbbVie, AstraZeneca, Biogen,

879 Celgene, Genentech, GSK, MSD, Pfizer, and Sanofi). Following biobanks are

880 acknowledged for collecting the FinnGen project samples: Auria Biobank, THL Biobank,

881 Helsinki Biobank, Northern Finland Biobank Borealis, Finnish Clinical Biobank Tampere, 
882 Biobank of Eastern Finland, Central Finland Biobank, Finnish Red Cross Blood Service

883 Biobank. UKB analyses were conducted under application no. 31063.

\section{Author contributions}

885 A.G. conceptualized the study. A.G. and S.J. designed the analysis plan. S.J. performed 886 most of the analyses that were not part of the FinnGen GWAS pipeline. The manuscript

887 was written by S.J. and A.G. The phenotyping approach for FinnGen was mainly 888 developed by T.K. and A.H., and they also provided methodological support. J.K. 889 participated in genetic fine-mapping and provided methodological support. M.C., J.T.R.,

890 N.M., and K.E.S. provided methodological support. H.M.O. provided advice on the HLA 891 analyses. M.P. provided statistical support by helping develop the approach for 892 quantifying uncertainty via resampling and helping develop the shrinkage approach. All 893 authors participated in reviewing the manuscript. 


\section{Extended Data Figures}

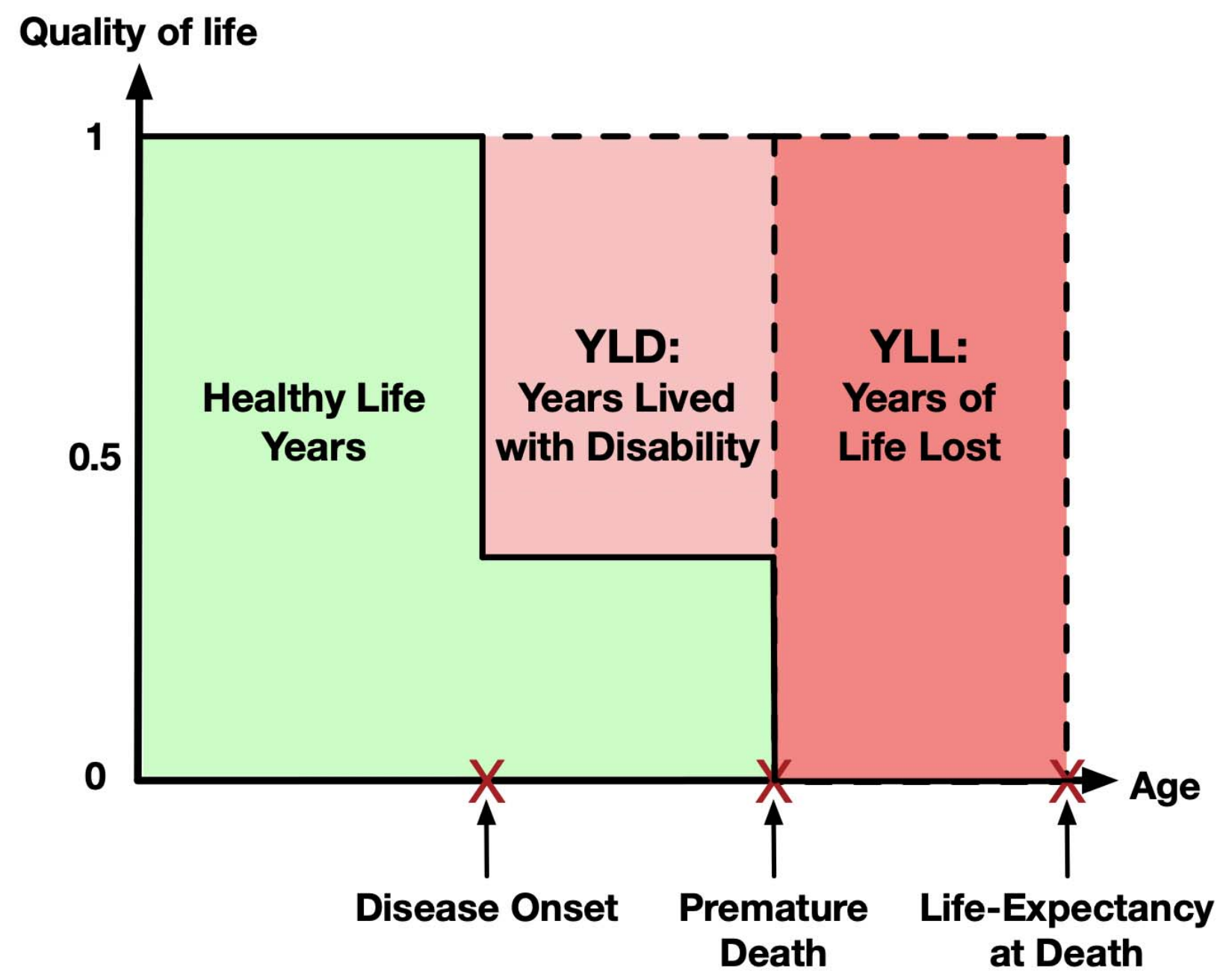

897 Extended Data Fig. 1: Schematic representation of how disability-adjusted life years (DALYs) 898 are constructed from years lived with disability (YLD), and years of life lost (YLL). DALYS are a 899 metric for measuring population-level disease burden that combines a measure of premature 900 mortality called years of life lost (YLL) and a measure of healthy life years lost due to lowered 901 quality of life called years lived with disability (YLD), so DALYs are the sum of YLDs and YLLs. 


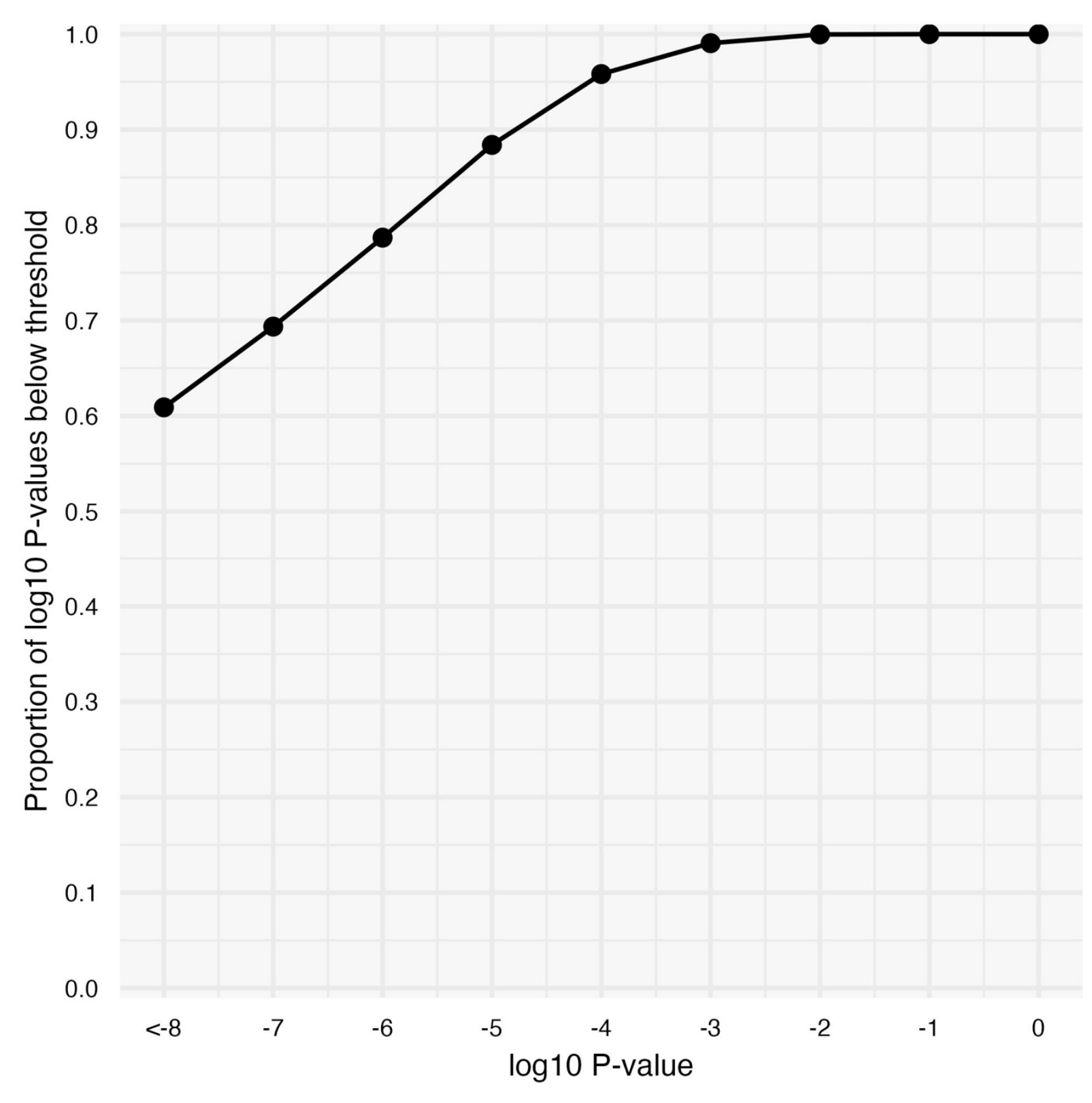

904 Extended Data Fig. 2: Cumulative distribution plot of $\log _{10} P$-values for all reported HRs

905 between genetic exposures and diseases $(n=3,123)$. $67.1 \%$ of the associations were genome-

906 wide significant $(\mathrm{P}<5 \times 10-8)$ and $99 \%$ had an association with $\mathrm{P}<7.3 \times 10-4$ 
medRxiv preprint doi: https://doi.org/10.1101/2022.01.25.22269831; this version posted January 28, 2022. The copyright holder for this preprint (which was not certified by peer review) is the author/funder, who has granted medRxiv a license to display the preprint in perpetuity.

It is made available under a CC-BY 4.0 International license.

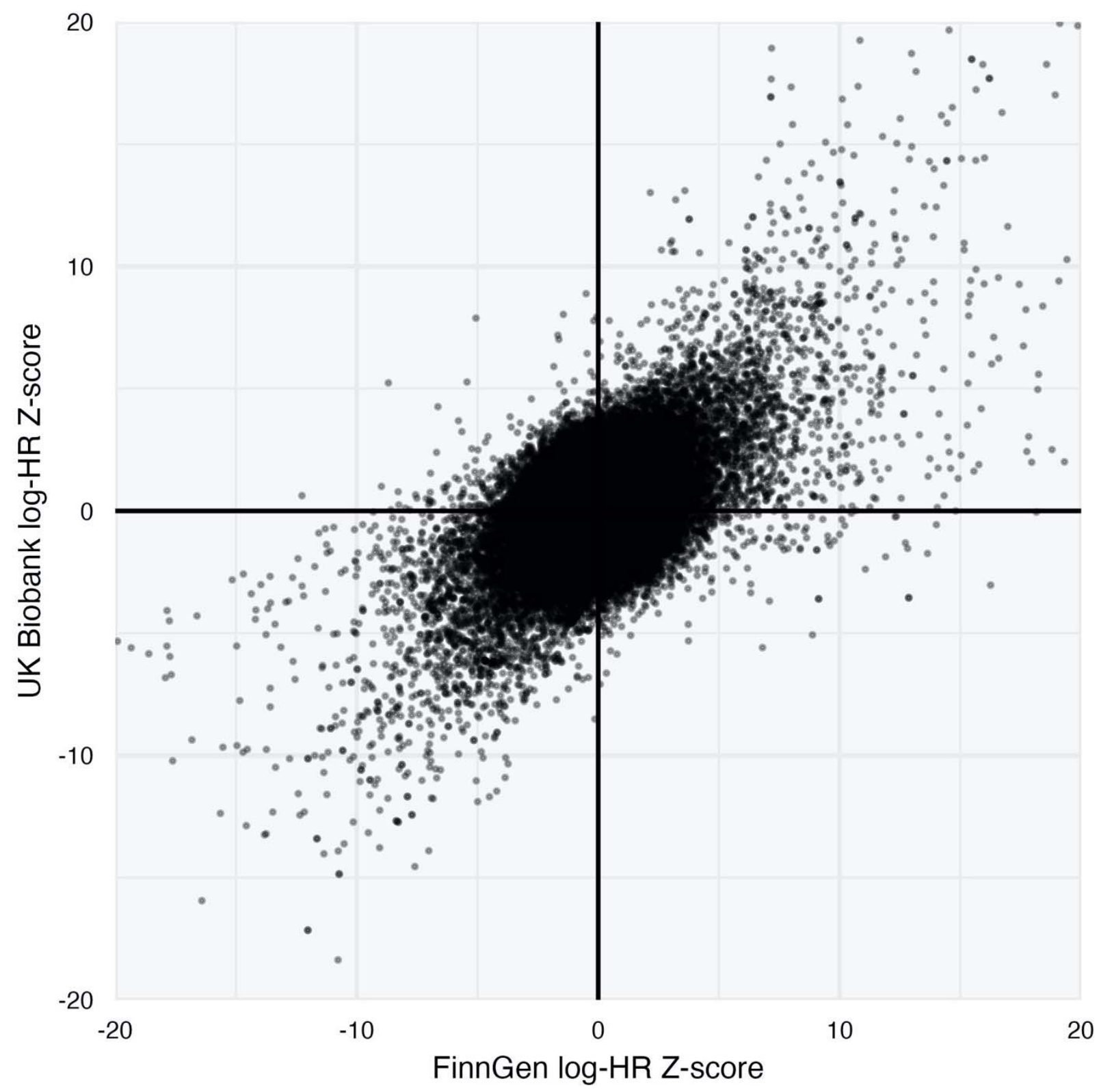

Extended Data Fig. 3: Comparison of effect sizes (log-HR Z-score) for common variant-disease

$910 \pm 20$, leaving out 36 points. 


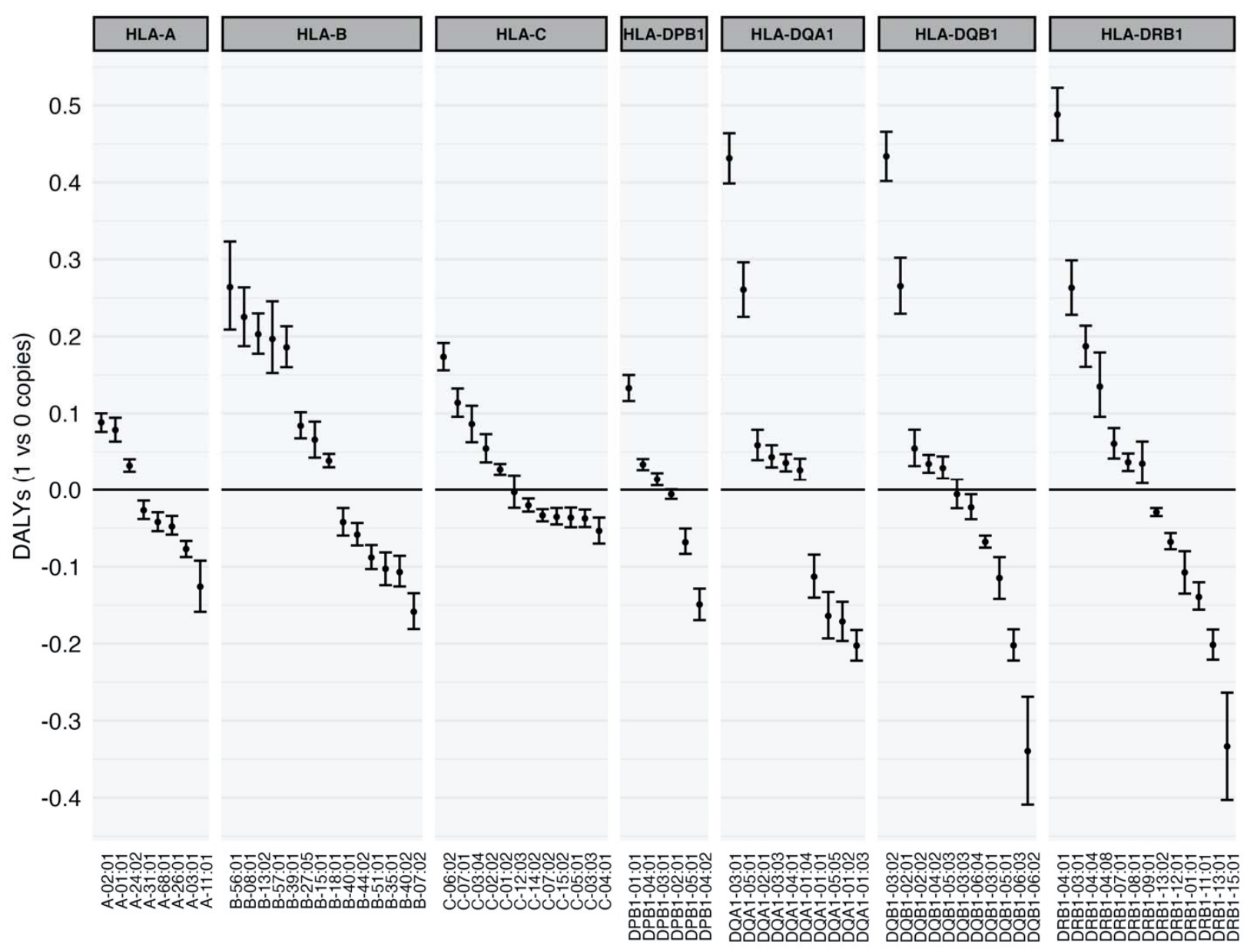
Extended Data Fig. 4: Effect of HLA alleles on DALYs by HLA gene. Note that each gene is

913 multiallelic, so the comparison of " 1 vs 0 copies" is more precisely the effect for having " 1 copy

914 of the allele in question and 1 average copy of other allele" vs "having 0 copies of the allele in

915 question and 2 average copies other alleles". See ST9 and Supplementary Information for

916 details. Error bars denote $95 \%$ confidence intervals. 
medRxiv preprint doi: https://doi.org/10.1101/2022.01.25.22269831; this version posted January 28, 2022. The copyright holder for this preprint (which was not certified by peer review) is the author/funder, who has granted medRxiv a license to display the preprint in perpetuity.

It is made available under a CC-BY 4.0 International license .

a

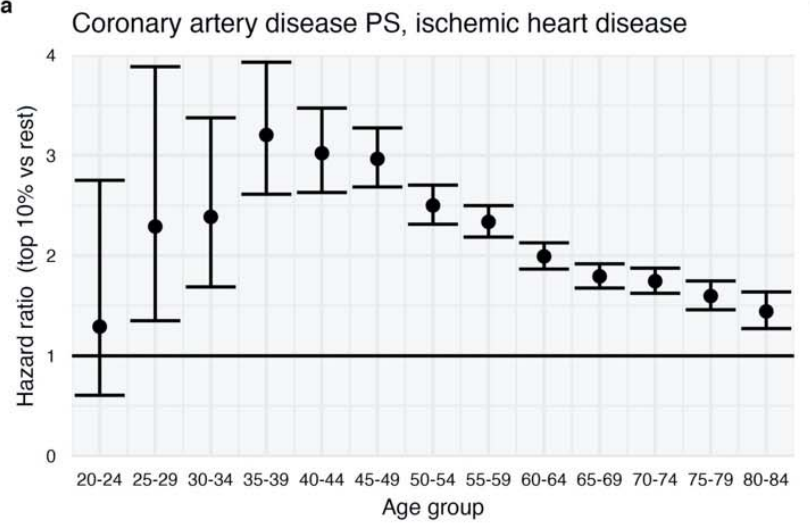

c

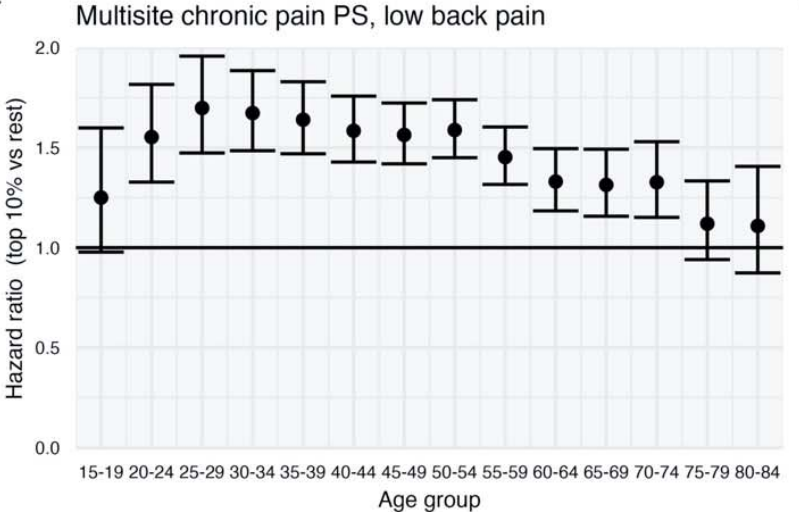

e rs11599804 (FGFR2), breast cancer

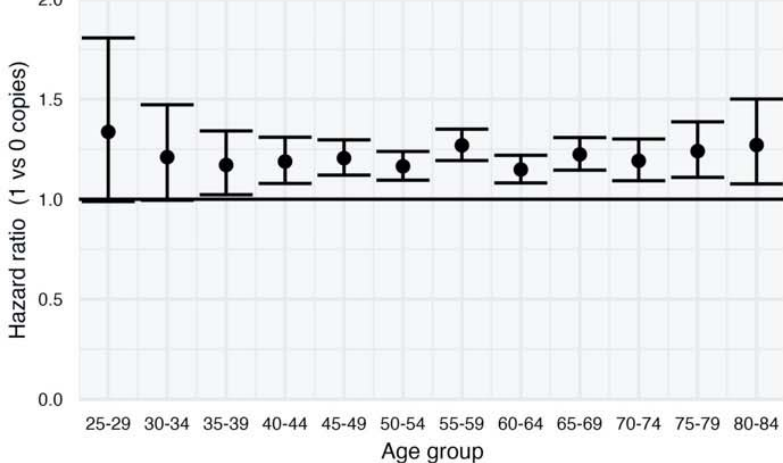

b $\quad$ rs3798220 (LPA), ischemic heart disease

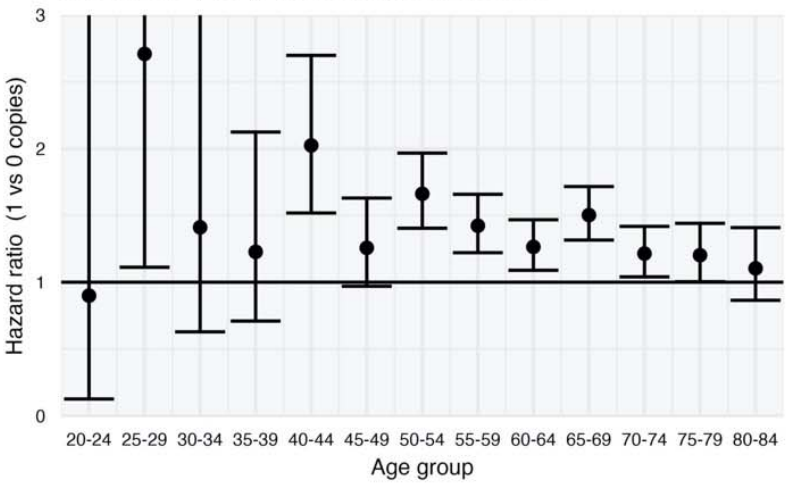

d rs429358 (APOE), Alzheimer's disease and other dementias

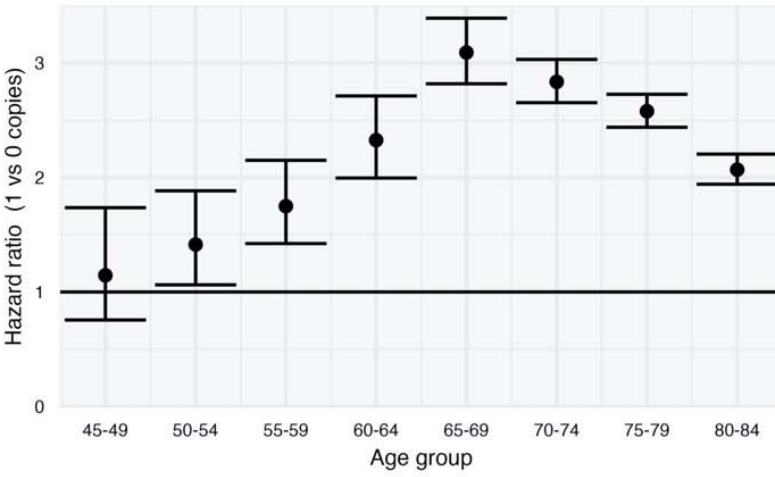
rs183373024 (POU5F1B), prostate cancer

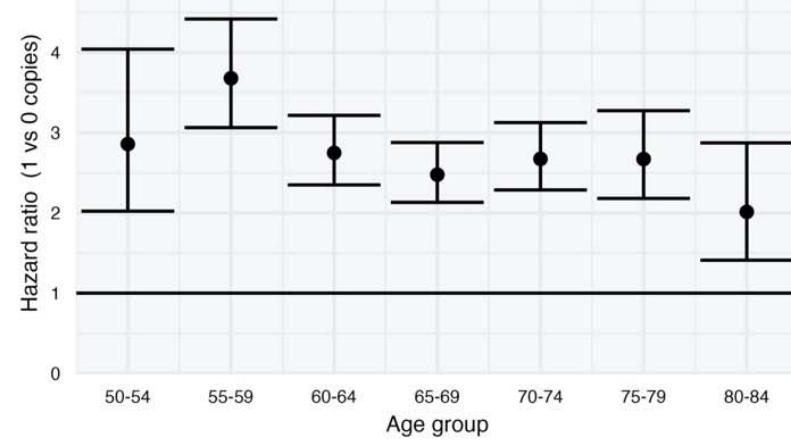

918 Extended Data Fig. 5: Hazard ratios by age group in FinnGen. For each age group we estimated

919 the HRs via a Cox proportional hazards model including individuals whose follow-up started

920 prior to beginning of the age interval that did not have a previous record of the disease. The

921 figure demonstrates age-varying HRs especially for ischemic heart disease, Alzheimer's disease

922 and other dementias, and low back pain $(\mathbf{a}, \mathbf{b}, \mathbf{c}, \mathbf{d})$. For breast cancer and prostate cancer, the

923 HRs were approximately constant across age groups $(\mathbf{e}, \mathbf{f})$. 
medRxiv preprint doi: https://doi.org/10.1101/2022.01.25.22269831; this version posted January 28, 2022. The copyright holder for this preprint (which was not certified by peer review) is the author/funder, who has granted medRxiv a license to display the preprint in perpetuity.

It is made available under a CC-BY 4.0 International license .

mean $=0.3, \mathrm{sd}=0.1, a=1, b=19$

mean $=0.3, s d=0.1, a=2, b=40$

mean $=0.3, s d=0.1, a=1, b=5$

mean $=0.6, s d=0.2, a=1, b=19$

mean $=0.6, \mathrm{sd}=0.2, \mathrm{a}=1, \mathrm{~b}=5$

mean $=0.6, s d=0.2, a=2, b=40$

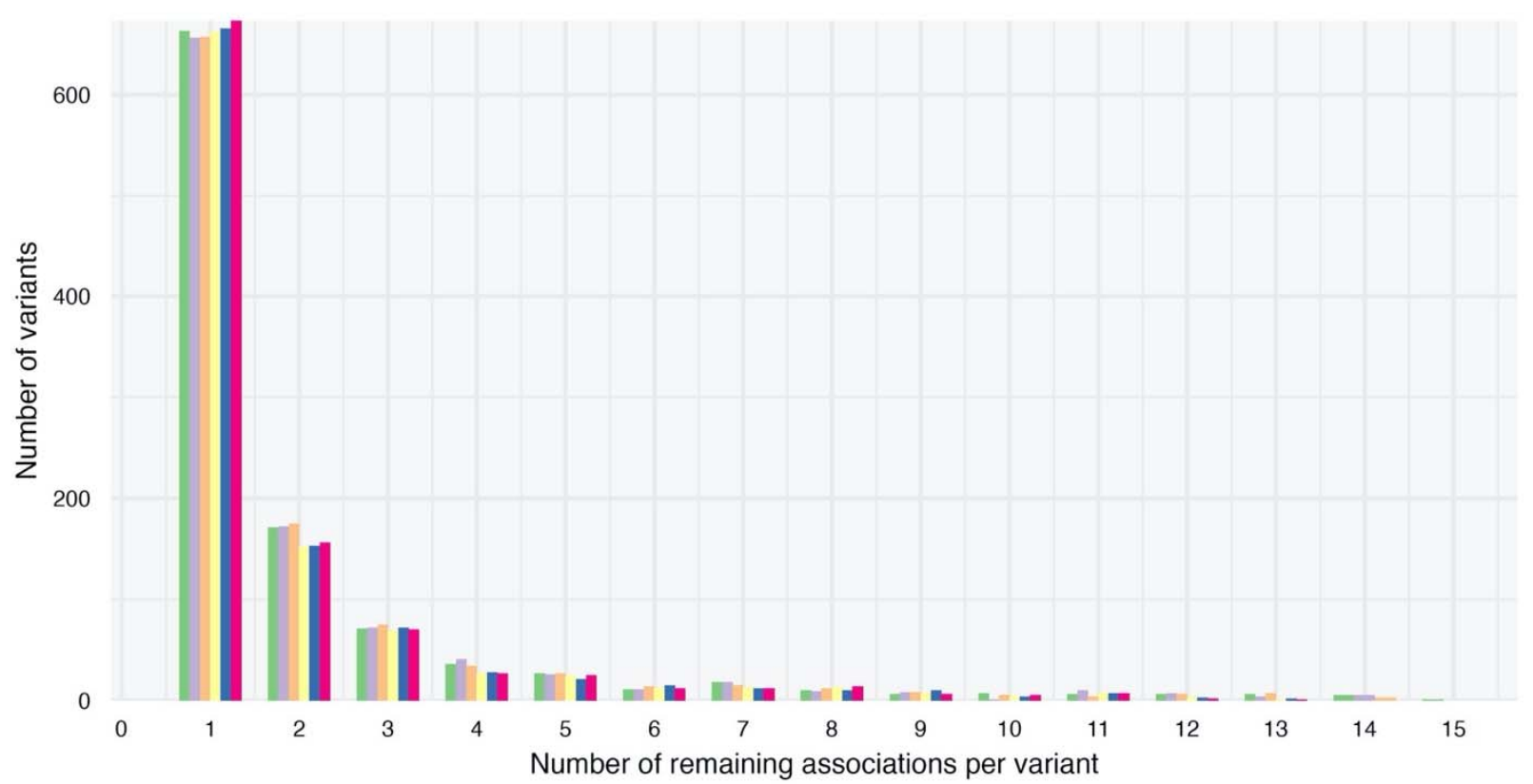

925 Extended Data Fig. 6: Histogram of the number of retained disease associations after

926 shrinkage using different prior parameters. In this plot we only consider common variants.

927 "mean" and "sd" correspond to the $\mu$ and $\sigma$ prior parameters, "a" and "b" correspond to the $\alpha$

928 and $\beta$ prior parameters (Methods). Overall, the distribution of retained association is not

929 sensitive to the choice of prior parameters. 


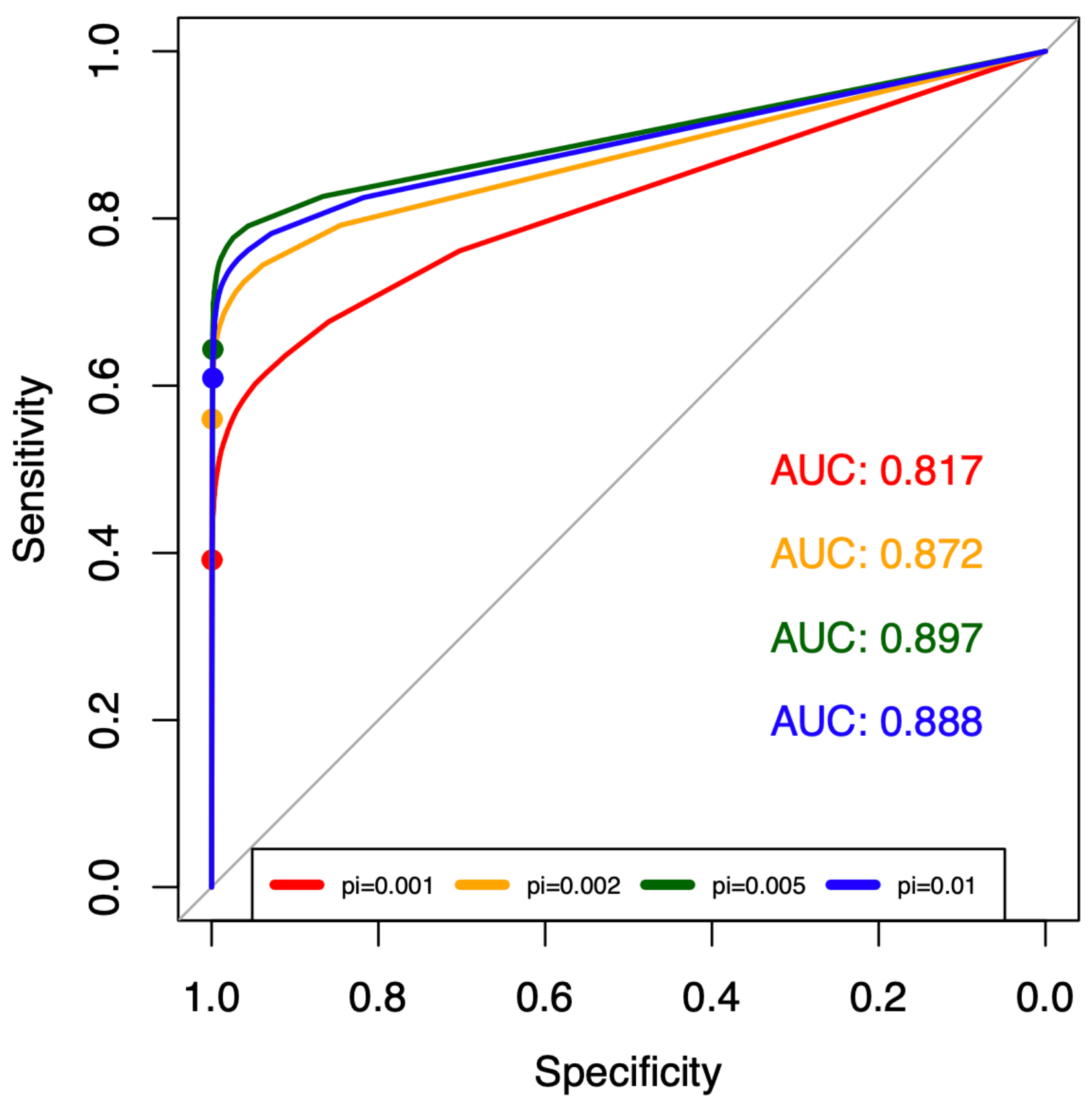

933 a classifier to identify true causal variants in simulated data. Using Hail, we simulated GWAS

934 summary statistics for 80 binary phenotypes with heritability sampled uniformly between 0.1

935 and 0.6 . We repeated this for four scenarios where the probability of a variant being causal (pi)

936 is $0.001,0.002,0.005$, and 0.01 (Supplementary Information). We then applied our shrinkage

937 procedure and classified those variants with posterior probability of being from the null model

938 of $<10 \%$ as being causal (colored dots on lines represent this threshold). See ST7 for details on 939 classification performance. 


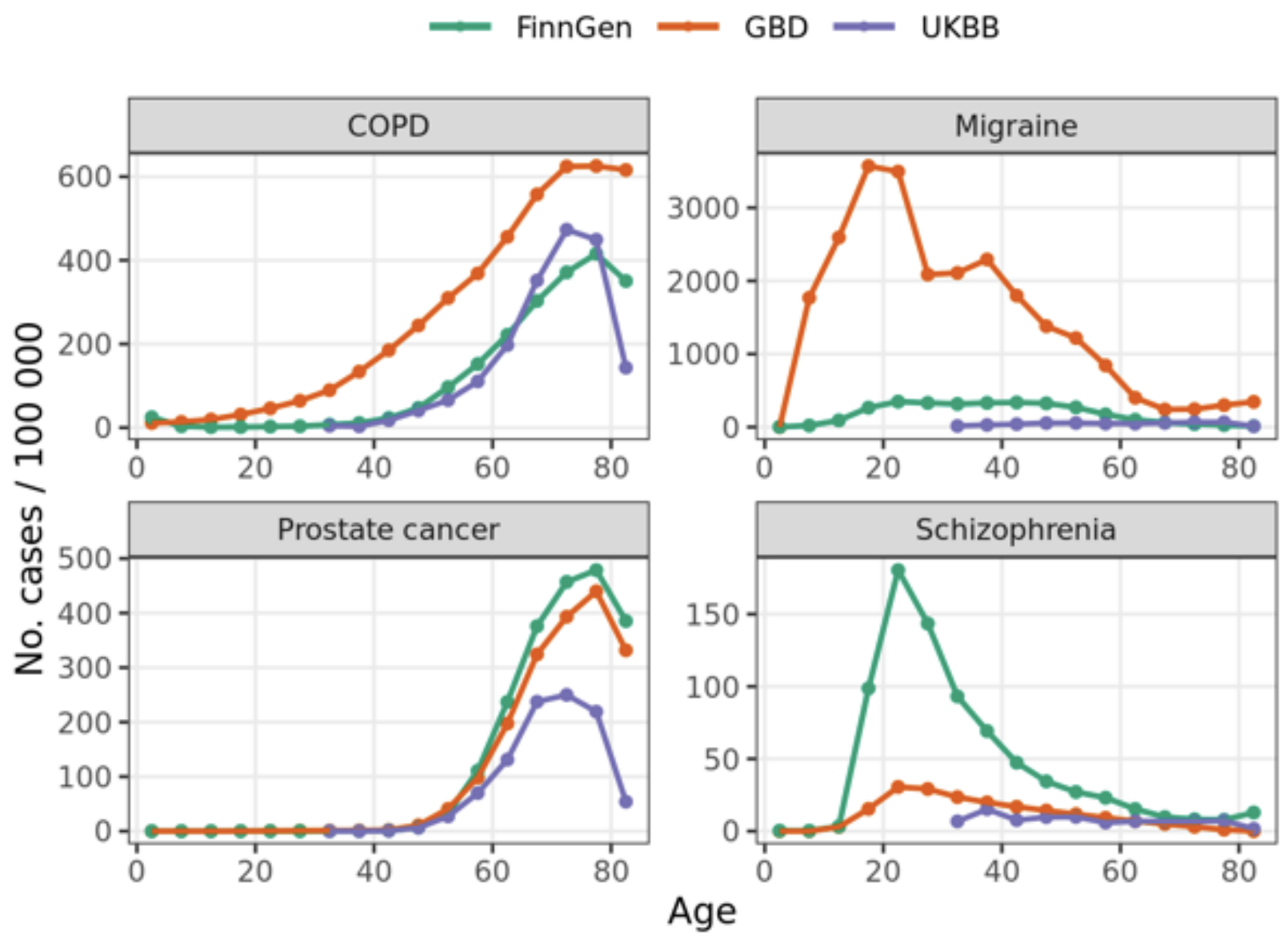

941 Extended data Fig. 8: 5-year incidence estimates of COPD, migraine, prostate cancer, and

942 schizophrenia in FinnGen and UK Biobank (UKBB) compared to the population incidence

943 estimates from the Global Burden of Disease Study 2019 for Finland 2019 (GBD). Number of

944 new cases per 100,000 was calculated as number of new cases per 100,000 during each age

945 interval divided by person-years contributed by individuals that were not cases in the earlier

946 age intervals. The incidence of some diseases like prostate cancer is well captured in UKB and

947 FinnGen, while other disease, such as migraine, as severely under-estimated. 